\title{
Explicit implied volatilities for multifactor local-stochastic volatility models
}

\author{
Matthew Lorig * $\quad$ Stefano Pagliarani ${ }^{\dagger} \quad$ Andrea Pascucci ${ }^{\ddagger}$
}

This version: December 1, 2014

\begin{abstract}
We consider an asset whose risk-neutral dynamics are described by a general class of local-stochastic volatility models and derive a family of asymptotic expansions for European-style option prices and implied volatilities. We also establish rigorous error estimates for these quantities. Our implied volatility expansions are explicit; they do not require any special functions nor do they require numerical integration. To illustrate the accuracy and versatility of our method, we implement it under four different model dynamics: CEV local volatility, Heston stochastic volatility, $3 / 2$ stochastic volatility, and SABR local-stochastic volatility.
\end{abstract}

Keywords: implied volatility, local-stochastic volatility, CEV, Heston, SABR.

\section{Introduction}

Local-stochastic volatility (LSV) models combine the features of local volatility (LV) and stochastic volatility (SV) models by describing the instantaneous volatility of an underlying $S$ by a function $\sigma\left(t, S_{t}, Y_{t}\right)$ where $Y$ is some auxiliary, possibly multidimensional, stochastic process (see, for instance, Lipton (2002), Alexander and Nogueira (2004), Ewald (2005), Henry-Labordere (2009) and Clark (2010)). Typically, unobservable LSV (or SV or LV) model parameters are obtained by calibrating to implied volatilities that are observed on the market. For this reason closed-form approximations for model-induced implied volatilities are useful. A number of different approaches have been taken for computing approximate implied volatilities in LV, SV and LSV models. We review some of these approaches below.

Concerning LV models, perhaps the earliest and most well-known implied volatility result is due to Hagan and Woodward (1999), who use singular perturbation methods to obtain an implied volatility expansion for general LV models. For certain models (e.g., CEV) they obtain closed-form approximations. More recently, Lorig (2013) uses regular perturbation methods to obtain an implied volatility expansion when a LV model can be written as a regular perturbation around Black-Scholes. Jacquier and Lorig (2013) extend and refine the results of Lorig (2013) to find closed-form approximations of implied volatility for local Lévy-type

\footnotetext{
*Department of Applied Mathematics, University of Washington, Seattle, USA. e-mail: mattlorig@gmail.com.

${ }^{\dagger}$ CMAP, Ecole Polytechnique Route de Saclay, 91128 Palaiseau Cedex, France. e-mail: stepagliara1@gmail.com. Work partially supported by the Chair Financial Risks of the Risk Foundation.

${ }^{\ddagger}$ Dipartimento di Matematica, Università di Bologna, Bologna, Italy. e-mail: andrea.pascucci@unibo.it
} 
models with jumps. Gatheral et al. (2012) examines the small-time asymptotics of implied volatility for LV models using heat kernel methods.

There is no shortage of implied volatility results for SV models either. Fouque et al. (2012) (see also Fouque et al. (2011) ) derive an asymptotic expansion for general multiscale stochastic volatility models using combined singular and regular perturbation theory. Forde and Jacquier (2011) use the Freidlin-Wentzell theory of large deviations for SDEs to obtain the small-time behavior of implied volatility for general stochastic volatility models with zero correlation. Their work adds mathematical rigor to previous work by Lewis (2007). Forde and Jacquier (2009) use large deviation techniques to obtain the small-time behavior of implied volatility in the Heston model (with correlation). They further refine these results in Forde et al. (2012). More recently, Jacquier and Lorig (2014) provide an explicit implied volatility approximation for any model with an analytically tractable characteristic function, which includes both affine stochastic volatility and exponential Lévy models.

Concerning LSV models, perhaps the most well-known implied volatility result is due to Hagan et al. (2002), who use WKB approximation methods to obtain implied volatility asymptotics in a LSV model with a CEV-like factor of local volatility and a GBM-like auxiliary factor of volatility (i.e., the SABR model). Another important contribution is due to Berestycki et al. (2004), who show that implied volatility in an LSV setting can be obtained by solving a quasi-linear parabolic partial differential equation. More recently, Henry-Labordère (2005) uses a heat kernel expansion on a Riemann manifold to derive first order asymptotics for implied volatility for any LSV model. As an example, he introduces the $\lambda$-SABR model, which is a LSV model with a mean reverting auxiliary factor of volatility, and obtains closed form asymptotic formulas for implied volatility in this setting. See also Henrv-Labordère (2009). Finally, we mention Watanabe (1987) and the recent work of Benhamou et al. (2010) and Bompis and Gobet (2012) who use Malliavin calculus techniques to derive closed-form approximations for implied volatility in an LSV setting. There are also some model-free results concerning the extreme-strike behavior of implied volatility. Most notably, we mention the work of Lee (2004) and Gao and Lee (2014).

In this paper, we provide closed-form approximations for implied volatility for a very general class of LSV models. We show (through a series of numerical experiments) that our approximation performs favorably when compared to other well-known implied volatility approximations (e.g., Hagan and Woodward (1999) for CEV, Forde et al. (2012) for Heston, and Hagan et al. (2002) for SABR). Additionally, we prove that our implied volatility expansion satisfies some rigorous error bounds for short-maturities. As a byproduct of the implied volatility analysis, we obtain some results concerning the short-maturity behavior of the Black-Scholes price, which appear to be new and of some independent interest. All of our results are consistent with the previously derived short-maturity asymptotic results appearing in Berestycki et al. (2002), Berestycki et al. (2004) and Bompis and Gobet (2012). The methodology presented in this paper builds upon the asymptotic pricing formulas first derived in Pagliarani and Pascucci (2012) for scalar diffusions and later extended in Pagliarani et al. (2013) and Lorig et al. (2014) for scalar Lévy-type processes.

The rest of this paper proceeds as follows: In Section 2, we introduce a general class of local-stochastic volatility models. We also derive a family of asymptotic expansions for European option prices and, under certain assumptions, provide rigorous error bounds for our pricing approximation. In Section 3 we translate our asymptotic price expansion into an asymptotic expansion of implied volatility. In Section 4 we establish rigorous error estimates for both our pricing and implied volatility expansions. Finally, in Section 5 we test our implied volatility approximation on four well-known models: CEV local volatility, Heston stochastic 
volatility, three-halves stochastic volatility and SABR local-stochastic volatility. Appendix $\mathrm{A}$ contains the results for the Black-Scholes price at short maturities.

\section{Asymptotic pricing for a general class of LSV models}

For simplicity, we assume a frictionless market, no arbitrage, zero interest rates and no dividends. We take, as given, an equivalent martingale measure $\mathbb{P}$, chosen by the market on a complete filtered probability space $\left(\Omega, \mathcal{F},\left\{\mathcal{F}_{t}, t \geq 0\right\}, \mathbb{P}\right)$. The filtration $\left\{\mathcal{F}_{t}, t \geq 0\right\}$ represents the history of the market. All stochastic processes defined below live on this probability space and all expectations are taken with respect to $\mathbb{P}$. We consider a strictly positive asset $S$ whose risk-neutral dynamics are given by $S=\exp (X)$ where $X=Z^{(1)}$ is the first component of a $d$-dimensional diffusion $Z=(X, Y)$, which solves

$$
\left.\begin{array}{rlrl}
\mathrm{d} Z_{t}^{(i)} & =\mu_{i}\left(t, Z_{t}\right) \mathrm{d} t+\sigma_{i}\left(t, Z_{t}\right) \mathrm{d} W_{t}^{(i)}, & & Z_{0}=z \in \mathbb{R}^{d}, \\
\mathrm{~d}\left\langle W^{(i)}, W^{(j)}\right\rangle_{t} & =\rho_{i j}\left(t, Z_{t}\right) \mathrm{d} t, & & \left|\rho_{i j}\right|<1 .
\end{array}\right\}
$$

We assume that SDE (2.1) has a unique strong solution. Sufficient conditions for the existence of a unique strong solution can be found, for example, in Ikeda and Watanabe (1989) or Pascucci (2011). We also assume that the coefficients are such that $\mathbb{E}\left[S_{t}\right]<\infty$ for all $t \in\left[0, T_{0}\right]$ for some positive $T_{0}$.

Let $V_{t}$ be the time $t$ value of a European derivative, expiring at time $T>t$ with payoff $\varphi\left(X_{T}\right)$. Using risk-neutral pricing, to value a European-style option we must compute functions of the form

$$
u(t, x, y):=\mathbb{E}\left[\varphi\left(X_{T}\right) \mid X_{t}=x, Y_{t}=y\right] .
$$

It is well-known that, under mild assumptions, the function $u$ satisfies the Kolmogorov backward equation

$$
\left(\partial_{t}+\mathcal{A}(t)\right) u(t, x, y)=0, \quad u(T, x, y)=\varphi(x),
$$

where the operator $\mathcal{A}(t)$ is given explicitly by

$$
\mathcal{A}(t)=\frac{1}{2} \sum_{i, j=1}^{d} \rho_{i j}(t, z) \sigma_{i}(t, z) \sigma_{j}(t, z) \partial_{z_{i} z_{j}}+\sum_{i=1}^{d} \mu_{i}(t, z) \partial_{z_{i}} .
$$

As a standing assumption, we impose $\mu_{1}=-\frac{1}{2} \sigma_{1}^{2}$ so as to ensure that $S=\mathrm{e}^{X}$ is a martingale. For many models in finance, the dimension of the diffusion is $d=1$ (e.g., CEV) or $d=2$ (e.g., Heston, SABR). For the special cases $d=1,2$, we write $\mathcal{A}(t)$ as

$$
\mathcal{A}(t)=a(t, x, y)\left(\partial_{x}^{2}-\partial_{x}\right)+f(t, x, y) \partial_{y}+b(t, x, y) \partial_{y}^{2}+c(t, x, y) \partial_{x} \partial_{y}, \quad(x, y) \in \mathbb{R}^{2}
$$

where

$$
a:=\frac{\sigma_{1}^{2}}{2}, \quad \quad f:=\mu_{2}, \quad c:=\frac{\sigma_{2}^{2}}{2}, \quad c:=\rho \sigma_{1} \sigma_{2} .
$$

When $d=1$ (i.e., local volatility models) only $a$ appears.

Remark 2.1 (Deterministic interest rates). For deterministic interest rates $r(t)$ one must compute expectations of the form

$$
\widetilde{u}(t, \widetilde{x}, y):=\mathbb{E}\left[\mathrm{e}^{-\int_{t}^{T} r(s) \mathrm{d} s} \varphi\left(\widetilde{X}_{T}\right) \mid \widetilde{X}_{t}=\widetilde{x}, Y_{t}=y\right], \quad \text { where } \quad \mathrm{d} \widetilde{X}_{t}=\mathrm{d} X_{t}+r(t) \mathrm{d} t .
$$


In this case a simple change of variables

$$
u(t, x(t, \widetilde{x}), y):=\mathrm{e}^{\int_{t}^{T} r(s)} \widetilde{u}(t, \widetilde{x}, y), \quad x(t, \widetilde{x}):=\widetilde{x}+\int_{t}^{T} r(s) \mathrm{d} s,
$$

reveals that the function $u$, as defined as in (2.5), satisfies (2.2).

\subsection{Polynomial expansions of $\mathcal{A}(t)$}

We note that (2.3) is a special case of the more general $d$-dimensional second order differential operator

$$
\mathcal{A}(t)=\sum_{i, j=1}^{d} a_{i j}(t, z) \partial_{z_{i} z_{j}}+\sum_{i=1}^{d} a_{i}(t, z) \partial_{z_{i}}, \quad t \in \mathbb{R}_{+}, z \in \mathbb{R}^{d} .
$$

Equivalently, we can also write the operator $\mathcal{A}(t)$ in a more compact form, i.e.

$$
\mathcal{A}(t):=\sum_{|\alpha| \leq 2} a_{\alpha}(t, z) D_{z}^{\alpha}, \quad t \in \mathbb{R}_{+}, z \in \mathbb{R}^{d}
$$

where, using standard multi-index notation we have

$$
\alpha=\left(\alpha_{1}, \cdots, \alpha_{d}\right) \in \mathbb{N}_{0}^{d}, \quad|\alpha|=\sum_{i=1}^{d} \alpha_{i}, \quad D_{z}^{\alpha}=\partial_{z_{1}}^{\alpha_{1}} \cdots \partial_{z_{d}}^{\alpha_{d}},
$$

In this section we introduce a family of expansion schemes for $\mathcal{A}(t)$, which we shall use to construct closedform approximate solutions (one for each family) of Cauchy problem (2.2).

Definition 2.2. Let $N \in \mathbb{N}_{0}$. We say that $\left(\mathcal{A}_{n}(t)\right)_{0 \leq n \leq N}$ is an $N$ th order polynomial expansion if

$$
\mathcal{A}_{n}(t, z) \equiv \mathcal{A}_{n}(t):=\sum_{|\alpha| \leq 2} a_{\alpha, n}(t, z) D_{z}^{\alpha}
$$

where

(i) for any $t \in[0, T]$ the functions $a_{\alpha, n}(t, \cdot)$ are polynomials, and for any $z \in \mathbb{R}^{d}$ the functions $a_{\alpha, n}(\cdot, z)$ belong to $L^{\infty}([0, T])$,

(ii) for any $t \in[0, T]$ we have $a_{\alpha, 0}(t, \cdot)=a_{\alpha, 0}(t)$, and the constant-in-space coefficients second order operator $\mathcal{A}_{0}(t)$ is elliptic.

The idea behind our approximation method is to choose a polynomial expansion such that the sequences of partial sums $\sum_{n=0}^{N} a_{\alpha, n}(t)$ approximate the coefficients $a_{\alpha}(t, z)$, either pointwise or in some norm. Below, we present some examples.

Example 2.3 (Taylor polynomial expansion). Assume the coefficients $a_{\alpha}(t, \cdot) \in C^{N}\left(\mathbb{R}^{d}\right)$. Then, for any fixed $\bar{z} \in \mathbb{R}^{d}, n \leq N$, we define $a_{\alpha, n}$ as the $n$-th order term of the Taylor expansion of $a_{\alpha}$ in the spatial variables around $\bar{z}$. That is, we set

$$
a_{\alpha, n}(\cdot, z)=\sum_{|\beta|=n} \frac{D_{z}^{\beta} a_{\alpha}(\cdot, \bar{z})}{\beta !}(z-\bar{z})^{\beta}, \quad n \leq N, \quad|\alpha| \leq 2,
$$

where as usual $\beta !=\beta_{1} ! \cdots \beta_{d} !$ and $z^{\beta}=z_{1}^{\beta_{1}} \cdots z_{d}^{\beta_{d}}$. 
Example 2.4 (Time-dependent Taylor polynomial expansion). Assume the coefficients $a_{\alpha}(t, \cdot) \in C^{N}\left(\mathbb{R}^{d}\right)$. Then, for any fixed $\bar{z}: \mathbb{R}_{+} \rightarrow \mathbb{R}^{d}$, we define $a_{\alpha, n}$ as the $n$-th order term of the Taylor expansion of $a_{\alpha}$ in the spatial variables around $\bar{z}(\cdot)$. That is, we set

$$
a_{\alpha, n}(\cdot, z)=\sum_{|\beta|=n} \frac{D_{z}^{\beta} a_{\alpha}(\cdot, \bar{z}(\cdot))}{\beta !}(z-\bar{z}(\cdot))^{\beta}, \quad n \leq N, \quad|\alpha| \leq 2 .
$$

Example 2.5 (Hermite polynomial expansion). Hermite expansions can be useful when the diffusion coefficients are not smooth. A remarkable example in financial mathematics is given by the Dupire's local volatility formula for models with jumps (see Friz et al. (2013)). In some cases, e.g., the well-known Variance-Gamma model, the fundamental solution (i.e., the transition density of the underlying stochastic model) has singularities. In such cases, it is natural to approximate it in some $L^{p}$ norm rather than in the pointwise sense. For the Hermite expansion centered at $\bar{z}$, one sets

$$
a_{\alpha, n}(t, z)=\sum_{|\beta|=n}\left\langle\mathbf{H}_{\beta}(\cdot-\bar{z}), a_{\alpha}(t, \cdot)\right\rangle_{\Gamma} \mathbf{H}_{\beta}(z-\bar{z}), \quad n \geq 0, \quad|\alpha| \leq 2 .
$$

where the inner product $\langle\cdot, \cdot\rangle_{\Gamma}$ is an integral over $\mathbb{R}^{d}$ with a Gaussian weighting centered at $\bar{z}$ and the functions $\mathbf{H}_{\beta}(z)=H_{\beta_{1}}\left(z_{1}\right) \cdots H_{\beta_{d}}\left(z_{d}\right)$ where $H_{n}$ is the $n$-th one-dimensional Hermite polynomial (properly normalized so that $\left\langle\mathbf{H}_{\alpha}, \mathbf{H}_{\beta}\right\rangle_{\Gamma}=\delta_{\alpha, \beta}$ with $\delta_{\alpha, \beta}$ being the Kronecker's delta function).

\subsection{Formal solution}

In this section, we introduce a heuristic procedure to construct an approximate solution of the backward Cauchy problem (2.2). Hereafter we will explicitly indicate $t$-dependence in all operators. On the other hand, we will generally hide $z$-dependence, except where it is needed for clarity.

Let us consider a polynomial expansion $\left(\mathcal{A}_{n}(t)\right)_{n \geq 0}$, and assume that the operator $\mathcal{A}(t)$ in (2.7) can be formally written as

$$
\mathcal{A}(t)=\mathcal{A}_{0}(t)+\mathcal{B}(t), \quad \mathcal{B}(t)=\sum_{n=1}^{\infty} \mathcal{A}_{n}(t)
$$

Inserting expansion (2.9) for $\mathcal{A}(t)$ into Cauchy problem (2.2) we find

$$
\left(\partial_{t}+\mathcal{A}_{0}(t)\right) u(t)=-\mathcal{B}(t) u(t), \quad u(T)=\varphi .
$$

By Duhamel's principle, we have

$$
u(t)=\mathcal{P}_{0}(t, T) \varphi+\int_{t}^{T} \mathrm{~d} t_{1} \mathcal{P}_{0}\left(t, t_{1}\right) \mathcal{B}\left(t_{1}\right) u\left(t_{1}\right),
$$

where $\mathcal{P}_{0}(t, T)$ is the semigroup of operators generated by $\mathcal{A}_{0}(t)$; we will explicitly define $\mathcal{P}_{0}(t, T)$ in (2.16). Inserting expression (2.10) for $u$ into the right-hand side of (2.10) and iterating we obtain

$$
\begin{aligned}
u\left(t_{0}\right)= & \mathcal{P}_{0}\left(t_{0}, T\right) \varphi+\int_{t_{0}}^{T} \mathrm{~d} t_{1} \mathcal{P}_{0}\left(t_{0}, t_{1}\right) \mathcal{B}\left(t_{1}\right) \mathcal{P}_{0}\left(t_{1}, T\right) \varphi \\
& \quad+\int_{t_{0}}^{T} \mathrm{~d} t_{1} \int_{t_{1}}^{T} \mathrm{~d} t_{2} \mathcal{P}_{0}\left(t_{0}, t_{1}\right) \mathcal{B}\left(t_{1}\right) \mathcal{P}_{0}\left(t_{1}, t_{2}\right) \mathcal{B}\left(t_{2}\right) u\left(t_{2}\right) \\
= & \cdots
\end{aligned}
$$




$$
\begin{aligned}
&= \mathcal{P}_{0}\left(t_{0}, T\right) \varphi+\sum_{k=1}^{\infty} \int_{t_{0}}^{T} \mathrm{~d} t_{1} \int_{t_{1}}^{T} \mathrm{~d} t_{2} \cdots \int_{t_{k-1}}^{T} \mathrm{~d} t_{k} \\
& \mathcal{P}_{0}\left(t_{0}, t_{1}\right) \mathcal{B}\left(t_{1}\right) \mathcal{P}_{0}\left(t_{1}, t_{2}\right) \mathcal{B}\left(t_{2}\right) \cdots \mathcal{P}_{0}\left(t_{k-1}, t_{k}\right) \mathcal{B}\left(t_{k}\right) \mathcal{P}_{0}\left(t_{k}, T\right) \varphi \\
&= \mathcal{P}_{0}\left(t_{0}, T\right) \varphi+\sum_{n=1}^{\infty} \sum_{k=1}^{n} \int_{t_{0}}^{T} \mathrm{~d} t_{1} \int_{t_{1}}^{T} \mathrm{~d} t_{2} \cdots \int_{t_{k-1}}^{T} \mathrm{~d} t_{k} \\
& \sum_{i \in I_{n, k}} \mathcal{P}_{0}\left(t_{0}, t_{1}\right) \mathcal{A}_{i_{1}}\left(t_{1}\right) \mathcal{P}_{0}\left(t_{1}, t_{2}\right) \mathcal{A}_{i_{2}}\left(t_{2}\right) \cdots \mathcal{P}_{0}\left(t_{k-1}, t_{k}\right) \mathcal{A}_{i_{k}}\left(t_{k}\right) \mathcal{P}_{0}\left(t_{k}, T\right) \varphi, \\
& I_{n, k}=\left\{i=\left(i_{1}, i_{2}, \cdots, i_{k}\right) \in \mathbb{N}^{k}: i_{1}+i_{2}+\cdots+i_{k}=n\right\} .
\end{aligned}
$$

To obtain (2.12) from (2.11) we have used the fact that from (2.9) the operator $\mathcal{B}(t)$ is an infinite sum, and we have partitioned on the sum $\left(i_{1}+i_{2}+\cdots+i_{k}\right)$ of the subscripts of the $\left(\mathcal{A}_{i_{k}}(t)\right)$. In light of expansion (2.12) we set

$$
u=\sum_{n=0}^{\infty} u_{n}
$$

where we have defined

$$
\begin{aligned}
u_{0}\left(t_{0}\right):= & \mathcal{P}_{0}\left(t_{0}, T\right) \varphi \\
u_{n}\left(t_{0}\right):= & \sum_{k=1}^{n} \int_{t_{0}}^{T} \mathrm{~d} t_{1} \int_{t_{1}}^{T} \mathrm{~d} t_{2} \cdots \int_{t_{k-1}}^{T} \mathrm{~d} t_{k} \\
& \sum_{i \in I_{n, k}} \mathcal{P}_{0}\left(t_{0}, t_{1}\right) \mathcal{A}_{i_{1}}\left(t_{1}\right) \mathcal{P}_{0}\left(t_{1}, t_{2}\right) \mathcal{A}_{i_{2}}\left(t_{2}\right) \cdots \mathcal{P}_{0}\left(t_{k-1}, t_{k}\right) \mathcal{A}_{i_{k}}\left(t_{k}\right) \mathcal{P}_{0}\left(t_{k}, T\right) \varphi .
\end{aligned}
$$

\subsection{Expression for $u_{0}$}

By assumption, the functions $a_{\alpha, 0}$ depend only on $t$. Therefore, the operator $\mathcal{A}_{0}(t)$ is the generator of a diffusion with time-dependent parameters. It will be useful to write the operator $\mathcal{A}_{0}(t)$ in the following form:

$$
\mathcal{A}_{0}(t)=\frac{1}{2} \sum_{i, j=1}^{d} C_{i j}(t) \partial_{z_{i} z_{j}}+\left\langle m(t), \nabla_{z}\right\rangle, \quad\left\langle m(t), \nabla_{z}\right\rangle=\sum_{i=1}^{d} m_{i}(t) \partial_{z_{i}} .
$$

Here the $d \times d$-matrix $C(t)$ is positive definite, for any $t \in[0, T]$, and $m$ is a $d$-dimensional vector. The action

of the semigroup of operators $\mathcal{P}_{0}\left(t_{0}, T\right)$ generated by $\mathcal{A}_{0}(t)$ is well-known. For any measurable function $\varphi$ that is at most exponentially growing we have

$$
u_{0}\left(t_{0}\right):=\mathcal{P}_{0}\left(t_{0}, T\right) \varphi=\int_{\mathbb{R}^{d}} \Gamma_{0}(t, \cdot ; T, \zeta) \varphi(\zeta) \mathrm{d} \zeta
$$

where $\Gamma_{0}(t, z ; T, \zeta)$ is the $d$-dimensional Gaussian density

$$
\Gamma_{0}(t, z ; T, \zeta)=\frac{1}{\sqrt{(2 \pi)^{d}|\mathbf{C}(t, T)|}} \exp \left(-\frac{1}{2}\left\langle\mathbf{C}^{-1}(t, T)(\zeta-z-\mathbf{m}(t, T)),(\zeta-z-\mathbf{m}(t, T))\right\rangle\right)
$$

with covariance matrix $\mathbf{C}(t, T)$ and mean vector $z+\mathbf{m}(t, T)$ given by:

$$
\mathbf{C}(t, T)=\int_{t}^{T} \mathrm{~d} s C(s), \quad \mathbf{m}(t, T)=\int_{t}^{T} \mathrm{~d} s m(s) .
$$

Note that the function $u_{0}$ as it is defined in (2.16) is the unique non-rapidly increasing solution of the homogeneous backward Cauchy problem $\left(\partial_{t}+\mathcal{A}_{0}(t)\right) u_{0}=0$ with terminal condition $u_{0}(T)=\varphi$. 


\subsection{Expression for $u_{n}$}

Remarkably, as the following theorem shows, every $u_{n}(t)$ can be expressed as a differential operator $\mathcal{L}_{n}(t, T)$ acting on $u_{0}(t)$.

Theorem 2.6. Assume $\varphi \in \mathcal{S}\left(\mathbb{R}^{d}\right)$, the Schwartz space of rapidly decreasing functions on $\mathbb{R}^{d}$. Then the function $u_{n}$ defined in (2.14) is given explicitly by

$$
u_{n}\left(t_{0}\right)=\mathcal{L}_{n}\left(t_{0}, T\right) u_{0}\left(t_{0}\right)
$$

where $u_{0}$ is given by (2.16) and

$$
\mathcal{L}_{n}\left(t_{0}, T\right)=\sum_{k=1}^{n} \int_{t_{0}}^{T} \mathrm{~d} t_{1} \int_{t_{1}}^{T} \mathrm{~d} t_{2} \cdots \int_{t_{k-1}}^{T} \mathrm{~d} t_{k} \sum_{i \in I_{n, k}} \mathcal{G}_{i_{1}}\left(t_{0}, t_{1}\right) \mathcal{G}_{i_{2}}\left(t_{0}, t_{2}\right) \cdots \mathcal{G}_{i_{k}}\left(t_{0}, t_{k}\right),
$$

with $I_{n, k}$ as defined in (2.13),

$$
\mathcal{G}_{i}\left(t_{0}, t_{k}\right):=\mathcal{A}_{i}\left(t_{k}, \mathcal{M}\left(t_{0}, t_{k}\right)\right)=\sum_{|\alpha| \leq 2} a_{\alpha, i}\left(t_{k}, \mathcal{M}\left(t_{0}, t_{k}\right)\right) D_{z}^{\alpha},
$$

with $\mathcal{A}_{i}(t, z)$ as in (2.8), and

$$
\mathcal{M}(t, s):=z+\mathbf{m}(t, s)+\mathbf{C}(t, s) \nabla_{z} .
$$

Proof. The main idea of the proof is to show that the operator $\mathcal{G}_{i}\left(t_{0}, t_{k}\right)$ in (2.19) satisfies

$$
\mathcal{P}_{0}\left(t_{0}, t_{k}\right) \mathcal{A}_{i}\left(t_{k}\right)=\mathcal{G}_{i}\left(t_{0}, t_{k}\right) \mathcal{P}_{0}\left(t_{0}, t_{k}\right) .
$$

Assuming (2.21) holds, we can use the fact that $\mathcal{P}_{0}\left(t_{k}, t_{k+1}\right)$ is a semigroup

$$
\mathcal{P}_{0}\left(t_{0}, T\right)=\mathcal{P}_{0}\left(t_{0}, t_{1}\right) \mathcal{P}_{0}\left(t_{1}, t_{2}\right) \cdots \mathcal{P}_{0}\left(t_{k-1}, t_{k}\right) \mathcal{P}_{0}\left(t_{k}, T\right),
$$

and we can re-write (2.14) as

$$
u_{n}\left(t_{0}\right)=\sum_{k=1}^{n} \int_{t_{0}}^{T} \mathrm{~d} t_{1} \int_{t_{1}}^{T} \mathrm{~d} t_{2} \cdots \int_{t_{k-1}}^{T} \mathrm{~d} t_{k} \sum_{i \in I_{n, k}} \mathcal{G}_{i_{1}}\left(t_{0}, t_{1}\right) \mathcal{G}_{i_{2}}\left(t_{0}, t_{2}\right) \cdots \mathcal{G}_{i_{k}}\left(t_{0}, t_{k}\right) \mathcal{P}_{0}\left(t_{0}, T\right) \varphi,
$$

from which (2.17)-(2.18) follows directly. Thus, we only need to show that $\mathcal{G}_{i}\left(t_{0}, t_{k}\right)$ satisfies (2.21). The condition $\varphi \in \mathcal{S}\left(\mathbb{R}^{d}\right)$ guarantees that $u_{0}(t, \cdot)$ belongs to the Schwartz class of rapidly decaying functions for all $t<T$. Therefore, any function of the form $p(z) D_{z}^{\beta} u_{0}(t, z)$, where $p$ is a polynomial, has a Fourier representation. Thus, without loss of generality, we can investigate how the operator $\mathcal{P}_{0}\left(t_{0}, t_{k}\right) \mathcal{A}_{i}\left(t_{k}\right)$ acts on the oscillating exponential $\mathrm{e}_{\lambda}(x):=\mathrm{e}^{\mathrm{i}\langle\lambda, x\rangle}$. We note that

$$
\mathcal{P}_{0}\left(t_{0}, t_{k}\right) \mathrm{e}_{\lambda}(z)=\mathrm{e}^{\Phi_{0}\left(t_{0}, t_{k}, \lambda\right)} \mathrm{e}_{\lambda}(z), \quad \text { where } \quad \Phi_{0}\left(t_{0}, t_{k}, \lambda\right)=\sum_{|\alpha| \leq 2}(\mathrm{i} \lambda)^{\alpha} \int_{t_{0}}^{t_{k}} \mathrm{~d} t a_{\alpha, 0}(t) .
$$

Next, we observe that the operator $\mathcal{M}_{i}\left(t_{0}, t_{k}\right)$, the $i$-th component of $\mathcal{M}\left(t_{0}, t_{k}\right)$ in (2.20) can be written

$$
\mathcal{M}_{i}\left(t_{0}, t_{k}\right)=M_{i}\left(t_{0}, t_{k},-i \nabla_{z}\right), \quad M_{i}\left(t_{0}, t_{k}, \lambda\right)=-i \partial_{\lambda_{i}}\left(\Phi_{0}\left(t_{0}, t_{k}, \lambda\right)+i\langle\lambda, z\rangle\right) .
$$

Using (2.23) we observe that for any natural number $n$ we have

$$
\left(-\mathrm{i} \partial_{\lambda_{i}}\right)^{n} \mathrm{e}^{\Phi_{0}\left(t_{0}, t_{k}, \lambda\right)} \mathrm{e}_{\lambda}(z)=\left(-\mathrm{i} \partial_{\lambda_{i}}\right)^{n-1} M_{i}\left(t_{0}, t_{k}, \lambda\right) \mathrm{e}^{\Phi_{0}\left(t_{0}, t_{k}, \lambda\right)} \mathrm{e}_{\lambda}(z)
$$




$$
\begin{aligned}
& =\mathcal{M}_{i}\left(t_{0}, t_{k}\right)\left(-\mathrm{i} \partial_{\lambda_{i}}\right)^{n-1} \mathrm{e}^{\Phi_{0}\left(t_{0}, t_{k}, \lambda\right)} \mathrm{e}_{\lambda}(z) \\
& =\cdots \\
& =\left[\mathcal{M}_{i}\left(t_{0}, t_{k}\right)\right]^{n} \mathrm{e}^{\Phi_{0}\left(t_{0}, t_{k}, \lambda\right)} \mathrm{e}_{\lambda}(z)
\end{aligned}
$$

Noting the $\partial_{\lambda_{i}}$ and $\partial_{\lambda_{j}}$ commute, it is clear that $\mathcal{M}_{i}\left(t_{0}, t_{k}\right)$ and $\mathcal{M}_{j}\left(t_{0}, t_{k}\right)$ also commute. Thus, for any multi-index $\beta$ we have

$$
\left(-i \nabla_{\lambda}\right)^{\beta} \mathrm{e}^{\Phi_{0}\left(t_{0}, t_{k}, \lambda\right)} \mathrm{e}_{\lambda}(z)=\left(\mathcal{M}\left(t_{0}, t_{k}\right)\right)^{\beta} \mathrm{e}^{\Phi_{0}\left(t_{0}, t_{k}, \lambda\right)} \mathrm{e}_{\lambda}(z),
$$

Finally, we compute

$$
\begin{aligned}
\mathcal{P}_{0}\left(t_{0}, t_{k}\right) \mathcal{A}_{i}\left(t_{k}\right) \mathrm{e}_{\lambda}(z) & =\sum_{|\alpha| \leq 2} \mathcal{P}_{0}\left(t_{0}, t_{k}\right) a_{\alpha, i}\left(t_{k}, z\right) D_{z}^{\alpha} \mathrm{e}_{\lambda}(z) \\
& =\sum_{|\alpha| \leq 2}(i \lambda)^{\alpha} \mathcal{P}_{0}\left(t_{0}, t_{k}\right) a_{\alpha, i}\left(t_{k}, z\right) \mathrm{e}_{\lambda}(z) \\
& =\sum_{|\alpha| \leq 2}(i \lambda)^{\alpha} a_{\alpha, i}\left(t_{k},-i \nabla_{\lambda}\right) \mathcal{P}_{0}\left(t_{0}, t_{k}\right) \mathrm{e}_{\lambda}(z) \\
& =\sum_{|\alpha| \leq 2}(i \lambda)^{\alpha} a_{\alpha, i}\left(t_{k},-i \nabla_{\lambda}\right) \mathrm{e}^{\Phi_{0}\left(t_{0}, t_{k}, \lambda\right)} \mathrm{e}_{\lambda}(z) \\
& =\sum_{|\alpha| \leq 2}(i \lambda)^{\alpha} a_{\alpha, i}\left(t_{k}, \mathcal{M}\left(t_{0}, t_{k}\right)\right) \mathrm{e}^{\Phi_{0}\left(t_{0}, t_{k}, \lambda\right)} \mathrm{e}_{\lambda}(z)
\end{aligned}
$$

which concludes the proof.

Remark 2.7 (Call payoffs). As we will show in Section 4.1 the functions $\left(u_{n}\right)$ can be alternatively characterized as solutions of a nested sequence of Cauchy problems (see equation (4.16) for the case when $\left(\mathcal{A}_{n}(t)\right)$ is expanded in a Taylor series as in Example 2.3). One can check directly that when $\varphi(x)=\left(\mathrm{e}^{x}-\mathrm{e}^{k}\right)$, the functions $\left(u_{n}\right)$ with each $u_{n}$ given by $u_{n}(t)=\mathcal{L}_{n}(t, T) u_{0}(t)$ satisfy the nested Cauchy problems. Thus, Theorem 2.6 also holds for Call option payoffs. This is true for any expansion $\left(\mathcal{A}_{n}(t)\right)$ satisfying Definition 2.2 ,

Remark 2.8. The number of terms in $\mathcal{L}_{n}(t, T)$ grows faster than $n$ !, which presents a computational challenge for large $n$. Nevertheless, we shall see in the numerical example provided in Section 5 that excellent approximations can be achieved with $n=3$.

Remark 2.9. When $d=1,2$, the operator $\mathcal{A}(t)$ is given by (2.4). In this case, we write $\mathcal{A}_{i}(t)$ as

$$
\mathcal{A}_{i}(t):=a_{i}(t, x, y)\left(\partial_{x}^{2}-\partial_{x}\right)+f_{i}(t, x, y) \partial_{y}+b_{i}(t, x, y) \partial_{y}^{2}+c_{i}(t, x, y) \partial_{x} \partial_{y},
$$

and we have explicitly

$$
\mathcal{G}_{i}(t, s):=a_{i}\left(s, \mathcal{M}_{x}(t, s), \mathcal{M}_{y}(t, s)\right)\left(\partial_{x}^{2}-\partial_{x}\right)+f_{i}\left(s, \mathcal{M}_{x}(t, s), \mathcal{M}_{y}(t, s)\right) \partial_{y}
$$




$$
\begin{gathered}
+b_{i}\left(s, \mathcal{M}_{x}(t, s), \mathcal{M}_{y}(t, s)\right) \partial_{y}^{2}+c_{i}\left(s, \mathcal{M}_{x}(t, s), \mathcal{M}_{y}(t, s)\right) \partial_{x} \partial_{y}, \\
\mathcal{M}_{x}(t, s)=x-\int_{t}^{s} \mathrm{~d} q a_{0}(q)+2 \int_{t}^{s} \mathrm{~d} q a_{0}(q) \partial_{x}+\int_{t}^{s} \mathrm{~d} q c_{0}(q) \partial_{y}, \\
\mathcal{M}_{y}(t, s)=y+\int_{t}^{s} \mathrm{~d} q f_{0}(q)+2 \int_{t}^{s} \mathrm{~d} q b_{0}(q) \partial_{y}+\int_{t}^{s} \mathrm{~d} q c_{0}(q) \partial_{x} .
\end{gathered}
$$

\section{Implied volatility expansion}

In this section, we derive an explicit implied volatility approximation from the asymptotic pricing expansion developed in the previous section. To begin our analysis, we fix a multifactor LSV model for $X=\log S$ as in (2.1), a time $t$, a maturity date $T>t$, the initial values $\left(X_{t}, Y_{t}\right)=(x, y) \in \mathbb{R} \times \mathbb{R}^{d-1}$ and a Call option payoff $\varphi\left(X_{T}\right)=\left(\mathrm{e}^{X_{T}}-\mathrm{e}^{k}\right)^{+}$. Our goal is to find the implied volatility for this particular Call option. To ease notation, we will sometimes suppress the dependence on $(t, T, x, y, k)$. However, the reader should keep in mind that the implied volatility of the option under consideration does depend on $(t, T, x, y, k)$, even if this is not explicitly indicated. Below, we provide definitions of the Black-Scholes price and implied volatility, which will be fundamental throughout this section.

Definition 3.1. The Black-Scholes price $u^{\mathrm{BS}}$ is given by

$$
u^{\mathrm{BS}}(\sigma ; \tau, x, k):=\mathrm{e}^{x} \mathcal{N}\left(d_{+}\right)-\mathrm{e}^{k} \mathcal{N}\left(d_{-}\right), \quad d_{ \pm}:=\frac{1}{\sigma \sqrt{\tau}}\left(x-k \pm \frac{\sigma^{2} \tau}{2}\right), \quad \tau:=T-t,
$$

where $\mathcal{N}$ is the $\mathrm{CDF}$ of a standard normal random variable.

Remark 3.2. It follows from (2.16) that when $\varphi(x)=\left(\mathrm{e}^{x}-\mathrm{e}^{k}\right)^{+}$we have

$$
u_{0}(t, x)=u^{\mathrm{BS}}\left(\sigma_{0} ; T-t, x, k\right), \quad \text { where } \quad \sigma_{0}=\sqrt{\frac{2}{T-t} \int_{t}^{T} a_{0}(s) \mathrm{d} s}
$$

where $a_{0}=C_{1,1}$ as in (2.15), or according to the multi-index notation, $a_{0}=a_{(2,0, \ldots, 0), 0}$.

Definition 3.3. For fixed $(\tau, x, k)$, the implied volatility corresponding to a Call price $u \in\left(\left(\mathrm{e}^{x}-\mathrm{e}^{k}\right)^{+}, \mathrm{e}^{x}\right)$ is defined as the unique strictly positive real solution $\sigma$ of the equation

$$
u^{\mathrm{BS}}(\sigma ; \tau, x, k)=u
$$

\subsection{Formal derivation}

We present here a formal derivation of our implied volatility expansion, which is based on the price expansion presented in Section 2. Throughout this section $(t, T, x, k)$ are fixed and thus we use the short notation

$$
u^{\mathrm{BS}}(\sigma)=u^{\mathrm{BS}}(\sigma ; T-t, x, k)
$$

for the Black-Scholes price. Consider the family of approximate Call prices indexed by $\delta$

$$
u(\delta)=\sum_{n=0}^{N} \delta^{n} u_{n}=u^{\mathrm{BS}}\left(\sigma_{0}\right)+\sum_{n=1}^{N} \delta^{n} u_{n}, \quad \delta \in[0,1],
$$


with $\sigma_{0}$ as in (3.2) and the functions $u_{n}(t)=\mathcal{L}_{n}(t, T) u_{0}(t)$ as given in Theorem 2.6. Note that setting $\delta=1$ yields our price expansion. Defining

$$
g(\delta):=\left(u^{\mathrm{BS}}\right)^{-1}(u(\delta)), \quad \delta \in[0,1] .
$$

we seek the implied volatility $\sigma=g(1)$. We will show in Section 4.2, Lemma 4.13, that under suitable assumptions $u(\delta) \in\left(\left(\mathrm{e}^{x}-\mathrm{e}^{k}\right)^{+}, \mathrm{e}^{x}\right)$ for any $\delta \in[0,1]$. This guarantees that $g(\delta)$ in (3.5) exists. By expanding both sides of (3.5) as a Taylor series in $\delta$, we see that $\sigma$ admits an expansion of the form

$$
\sigma=g(1)=\sigma_{0}+\sum_{n=1}^{\infty} \sigma_{n}, \quad \sigma_{n}=\left.\frac{1}{n !} \partial_{\delta}^{n} g(\delta)\right|_{\delta=0}
$$

Note that, by (3.4) we also have

$$
u_{n}=\left.\frac{1}{n !} \partial_{\delta}^{n} u^{\mathrm{BS}}(g(\delta))\right|_{\delta=0}, \quad 1 \leq n \leq N .
$$

The right-hand side of (3.7) can be computed by applying the Bell polynomial version of the Faa di Bruno's formula, which is given in Appendix B,

$$
u_{n}=\left.\frac{1}{n !} \sum_{h=1}^{n} \partial_{\sigma}^{h} u^{\mathrm{BS}}\left(\sigma_{0}\right) \mathbf{B}_{n, h}\left(\partial_{\delta} g(\delta), \partial_{\delta}^{2} g(\delta), \ldots, \partial_{\delta}^{n-h+1} g(\delta)\right)\right|_{\delta=0}, \quad 1 \leq n \leq N .
$$

Combining (3.8) with (3.6), one can solve for $\sigma_{n}$ explicitly in terms of $\left(\sigma_{k}\right)_{0 \leq k \leq n-1}$, which yields

$$
\sigma_{n}=\frac{u_{n}}{\partial_{\sigma} u^{\mathrm{BS}}\left(\sigma_{0}\right)}-\frac{1}{n !} \sum_{h=2}^{n} \mathbf{B}_{n, h}\left(1 ! \sigma_{1}, 2 ! \sigma_{2}, \ldots,(n-h+1) ! \sigma_{n-h+1}\right) \frac{\partial_{\sigma}^{h} u^{\mathrm{BS}}\left(\sigma_{0}\right)}{\partial_{\sigma} u^{\mathrm{BS}}\left(\sigma_{0}\right)}, \quad 1 \leq n \leq N .
$$

Note that expression (3.9) for $\sigma_{n}$ involves two sorts of terms: $u_{n} / \partial_{\sigma} u^{\mathrm{BS}}\left(\sigma_{0}\right)$ and $\partial_{\sigma}^{n} u^{\mathrm{BS}}\left(\sigma_{0}\right) / \partial_{\sigma} u^{\mathrm{BS}}\left(\sigma_{0}\right)$. We will prove that these terms can be computed explicitly without any numerical integration or special functions. The proof will rely on the following lemma.

Lemma 3.4. Let $m \geq 0$ and fix $\left(t, T, k, \sigma_{0}\right)$. Then

$$
\frac{\partial_{x}^{m}\left(\partial_{x}^{2}-\partial_{x}\right) u^{\mathrm{BS}}\left(\sigma_{0}\right)}{\left(\partial_{x}^{2}-\partial_{x}\right) u^{\mathrm{BS}}\left(\sigma_{0}\right)}=\left(-\frac{1}{\sigma_{0} \sqrt{2 \tau}}\right)^{m} H_{m}(\zeta), \quad \zeta:=\frac{x-k-\frac{1}{2} \sigma_{0}^{2} \tau}{\sigma_{0} \sqrt{2 \tau}}, \quad \tau:=T-t,
$$

where $H_{n}(\zeta):=(-1)^{n} \mathrm{e}^{\zeta^{2}} \partial_{\zeta}^{n} \mathrm{e}^{-\zeta^{2}}$ is the $n$-th Hermite polynomial.

Proof. Using the Black-Scholes formula (3.1), a direct computation shows

$$
\left(\partial_{x}^{2}-\partial_{x}\right) u^{\mathrm{BS}}\left(\sigma_{0}\right)=\frac{1}{\sigma_{0} \sqrt{2 \pi \tau}} \mathrm{e}^{-\zeta^{2}+k}
$$

with $\zeta=\zeta(x)$ as above. Hence

$$
\frac{\partial_{x}^{m}\left(\partial_{x}^{2}-\partial_{x}\right) u^{\mathrm{BS}}\left(\sigma_{0}\right)}{\left(\partial_{x}^{2}-\partial_{x}\right) u^{\mathrm{BS}}\left(\sigma_{0}\right)}=\mathrm{e}^{\zeta^{2}} \partial_{x}^{m} \mathrm{e}^{-\zeta^{2}}=\left(\frac{1}{\sigma_{0} \sqrt{2 \tau}}\right)^{m} \mathrm{e}^{z^{2}} \partial_{\zeta}^{m} \mathrm{e}^{-\zeta^{2}}=\left(\frac{-1}{\sigma_{0} \sqrt{2 \tau}}\right)^{m} H_{m}(\zeta),
$$

where in the last equality we have used the definition of the $m$ th Hermite polynomial, recalled above.

Proposition 3.5. Fix $\left(t, T, k, \sigma_{0}\right)$ and let $\zeta$ and $\tau$ be as in Lemma 3.4. Then for any $n \geq 2$ we have

$$
\frac{\partial_{\sigma}^{n} u^{\mathrm{BS}}\left(\sigma_{0}\right)}{\partial_{\sigma} u^{\mathrm{BS}}\left(\sigma_{0}\right)}=\sum_{q=0}^{\lfloor n / 2\rfloor} \sum_{p=0}^{n-q-1} c_{n, n-2 q} \sigma_{0}^{n-2 q-1} \tau^{n-q-1}\left(\begin{array}{c}
n-q-1 \\
p
\end{array}\right)\left(-\frac{1}{\sigma_{0} \sqrt{2 \tau}}\right)^{p+n-q-1} H_{p+n-q-1}(\zeta),
$$

where the coefficients $\left(c_{n, n-2 k}\right)$ are defined recursively by

$$
c_{n, n}=1, \quad \text { and } \quad c_{n, n-2 q}=(n-2 q+1) c_{n-1, n-2 q+1}+c_{n-1, n-2 q-1}, \quad q \in\{1,2, \cdots,\lfloor n / 2\rfloor\} .
$$


Proof. Define the operator $\mathcal{J}:=\tau\left(\partial_{x}^{2}-\partial_{x}\right)$. It is classical that $\partial_{\sigma} u^{\mathrm{BS}}\left(\sigma_{0}\right)=\sigma_{0} \mathcal{J} u^{\mathrm{BS}}\left(\sigma_{0}\right)$. We claim that the following identity holds for any $n \in \mathbb{N}$

$$
\partial_{\sigma}^{n} u^{\mathrm{BS}}\left(\sigma_{0}\right)=\sum_{q=0}^{\lfloor n / 2\rfloor} c_{n, n-2 q} \sigma_{0}^{n-2 q} \mathrm{~g}^{n-q} u^{\mathrm{BS}}\left(\sigma_{0}\right)
$$

where $c_{n, n}=1$ and $c_{n, n-2 q}=(n-2 q+1) c_{n-1, n-2 q+1}+c_{n-1, n-2 q-1}$ for any integer $q \in\{1,2, \cdots,\lfloor n / 2\rfloor\}$. The proof of (3.11) is a simple yet tedious recursion relation, which we omit for brevity. Now, we compute

$$
\begin{aligned}
\frac{\partial_{\sigma}^{n} u^{\mathrm{BS}}\left(\sigma_{0}\right)}{\partial_{\sigma} u^{\mathrm{BS}}\left(\sigma_{0}\right)} & =\sum_{q=0}^{\lfloor n / 2\rfloor} c_{n, n-2 q} \sigma_{0}^{n-2 q} \frac{\partial^{n-q} u^{\mathrm{BS}}\left(\sigma_{0}\right)}{\partial_{\sigma} u^{\mathrm{BS}}\left(\sigma_{0}\right)}=\sum_{q=0}^{\lfloor n / 2\rfloor} c_{n, n-2 q} \sigma_{0}^{n-2 q} \tau^{n-q} \frac{\left(\partial_{x}^{2}-\partial_{x}\right)^{n-q} u^{\mathrm{BS}}\left(\sigma_{0}\right)}{\partial_{\sigma} u^{\mathrm{BS}}\left(\sigma_{0}\right)} \\
& =\sum_{q=0}^{\lfloor n / 2\rfloor} c_{n, n-2 q} \sigma_{0}^{n-2 q} \tau^{n-q} \frac{\left(\partial_{x}^{2}-\partial_{x}\right)^{n-q-1}\left(\partial_{x}^{2}-\partial_{x}\right) u^{\mathrm{BS}}\left(\sigma_{0}\right)}{\tau \sigma_{0}\left(\partial_{x}^{2}-\partial_{x}\right) u^{\mathrm{BS}}\left(\sigma_{0}\right)} \\
& =\sum_{q=0}^{\lfloor n / 2\rfloor} \sum_{p=0}^{n-q-1} c_{n, n-2 q} \sigma_{0}^{n-2 q-1} \tau^{n-q-1}\left(\begin{array}{c}
n-q-1 \\
p
\end{array}\right) \frac{\partial_{x}^{p+n-q-1}\left(\partial_{x}^{2}-\partial_{x}\right) u^{\mathrm{BS}}\left(\sigma_{0}\right)}{\left(\partial_{x}^{2}-\partial_{x}\right) u^{\mathrm{BS}}\left(\sigma_{0}\right)} \\
& =\sum_{q=0}^{\lfloor n / 2\rfloor} \sum_{p=0}^{n-q-1} c_{n, n-2 q} \sigma_{0}^{n-2 q-1} \tau^{n-q-1}\left(\begin{array}{c}
n-q-1 \\
p
\end{array}\right)\left(-\frac{1}{\sigma_{0} \sqrt{2 \tau}}\right)^{p+n-q-1} H_{p+n-q-1}(\zeta),
\end{aligned}
$$

where to obtain the last equality we have used (3.10).

Proposition 3.6. Fix $(t, T, x, y)$. For every polynomial expansion $\left(\mathcal{A}_{n}(t)\right)$ satisfying Definition 2.2 and for every $n \in \mathbb{N}$, the ratio $u_{n} / \partial_{\sigma} u^{\mathrm{BS}}\left(\sigma_{0}\right)$ is a finite sum of the form

$$
\frac{u_{n}}{\partial_{\sigma} u^{\mathrm{BS}}\left(\sigma_{0}\right)}=\sum_{m} \chi_{m}^{(n)}(t, T, x, y)\left(-\frac{1}{\sigma_{0} \sqrt{2 \tau}}\right)^{m} H_{m}(\zeta),
$$

where $\zeta$ and $\tau$ are as in Lemma 3.4. The coefficients $\chi_{m}^{(n)}(t, T, x, y)$ are explicit function of $x$ and $y$ and contain iterated integrals in the time-variable. If the iterated time-integrals can be computed explicitly then $\chi_{m}^{(n)}(t, T, x, y)$ is explicit in all variables.

Proof. From equation (2.25) and Remark 3.2 we observe that, for the case $d=1,2$

$$
\mathcal{G}_{i}(t, s) u_{0}:=a_{i}\left(s, \mathcal{M}_{x}(t, s), \mathcal{M}_{y}(t, s)\right)\left(\partial_{x}^{2}-\partial_{x}\right) u^{\mathrm{BS}}\left(\sigma_{0}\right) .
$$

For a general LSV model with $d-1$ factors of volatility we have

$$
\mathcal{M}_{y}(t, s)=\left(\mathcal{M}_{y_{1}}(t, s), \mathcal{M}_{y_{2}}(t, s), \ldots, \mathcal{M}_{y_{d-1}}(t, s)\right) .
$$

Therefore, using Theorem 2.6 we have

$$
\frac{u_{n}(t)}{\partial_{\sigma} u^{\mathrm{BS}}\left(\sigma_{0}\right)}=\frac{\mathcal{L}_{n}(t, T) u_{0}(t)}{\partial_{\sigma} u^{\mathrm{BS}}\left(\sigma_{0}\right)}=\frac{\widetilde{\mathcal{L}}_{n}(t, T)\left(\partial_{x}^{2}-\partial_{x}\right) u^{\mathrm{BS}}\left(\sigma_{0}\right)}{\tau \sigma_{0}\left(\partial_{x}^{2}-\partial_{x}\right) u^{\mathrm{BS}}\left(\sigma_{0}\right)},
$$

where

$$
\widetilde{\mathcal{L}}_{n}(t, T)=\sum_{k=1}^{n} \int_{t}^{T} \mathrm{~d} t_{1} \int_{t_{1}}^{T} \mathrm{~d} t_{2} \cdots \int_{t_{k-1}}^{T} \mathrm{~d} t_{k} \sum_{i \in I_{n, k}} \mathcal{G}_{i_{1}}\left(t, t_{1}\right) \cdots \mathcal{G}_{i_{k-1}}\left(t, t_{k-1}\right) a_{i_{k}}\left(s, \mathcal{M} \mathcal{M}_{x}\left(t, t_{k}\right), \mathcal{M}_{y}\left(t, t_{k}\right)\right) .
$$


It is clear that $\widetilde{\mathcal{L}}_{n}(t, T)$ is a differential operator that takes derivatives with respect to $x$ and $y$ and has coefficients that depend on $(t, T, x, y)$. Noting that $\partial_{y}^{m} u^{\mathrm{BS}}\left(\sigma_{0}\right)=0$ for all $m \geq 1$, it is clear from (3.13) that $u_{n} / \partial_{\sigma} u^{\mathrm{BS}}\left(\sigma_{0}\right)$ is of the form

$$
\frac{u_{n}(t)}{\partial_{\sigma} u^{\mathrm{BS}}\left(\sigma_{0}\right)}=\sum_{m} \chi_{m}^{(n)}(t, T, x, y) \frac{\partial_{x}^{m}\left(\partial_{x}^{2}-\partial_{x}\right) u^{\mathrm{BS}}\left(\sigma_{0}\right)}{\left(\partial_{x}^{2}-\partial_{x}\right) u^{\mathrm{BS}}\left(\sigma_{0}\right)} .
$$

Equation (3.12) follows from equation (3.15) and Lemma 3.4. The sequence of coefficients $\left(\chi_{m}^{(n)}\right)$ must be computed on a case-by-case basis because the $\left(\chi_{m}^{(n)}\right)$ depend on the coefficients of the generator $\mathcal{A}(t)$ and the choice of polynomial expansion $\left(\mathcal{A}_{n}(t)\right)$.

From Propositions 3.5 and 3.6 it is apparent that, as long as the iterated time integrals in (3.14) can be computed explicitly (which is always the case when the coefficients in the polynomial expansion $\left(\mathcal{A}_{n}(t)\right.$ ) are piece-wise polynomial in time), every term in (3.9) can be computed without the need for numerical integration or special functions.

Explicit expressions for each $\sigma_{n}$ in the sequence $\left(\sigma_{n}\right)_{n \geq 1}$ can be computed by hand. However, since the number of terms grows quickly with $n$, it is helpful to use a computer algebra program such as Wolfram's Mathematica. In Appendix [C, we provide explicit expressions for $\sigma_{n}$ for $n \leq 2$ the coefficients of $\mathcal{A}(t)$ are expanded as a Taylor series, as in Example 2.3. On the authors' website, we also provide Mathematica notebooks which contains the expressions for $\sigma_{n}$ for $n \leq 4$ for the LSV models described in Section 5 ,

Remark 3.7. When the risk-free rate of interest is a deterministic function of time $r(t)$, the implied volatility results above hold with $k \rightarrow k-\int_{t}^{T} r(s) \mathrm{d} s$.

\section{Asymptotic error estimates for Taylor expansions}

In this section we provide pointwise short-time error estimates for the approximate solution of Cauchy problem (2.2) discussed in Section 2, as well as for the approximate implied volatility presented in Section 3. Throughout this section we shall assume that $T_{0}>0$ and $N \in \mathbb{N}_{0}$ are fixed and the coefficients of the operator $\mathcal{A}(t)$ in (2.6) satisfy the following assumption:

Assumption 4.1. There exists a positive constant $M$ such that:

i) Uniform ellipticity:

$$
M^{-1}|\xi|^{2}<\sum_{i, j=1}^{d} a_{i j}(t, z) \xi_{i} \xi_{j}<M|\xi|^{2}, \quad t \in\left[0, T_{0}\right], z, \xi \in \mathbb{R}^{d}
$$

ii) Regularity and boundedness: the coefficients $a_{i j}, a_{i} \in C\left(\left[0, T_{0}\right] \times \mathbb{R}^{d}\right)$ and $a_{i j}(t, \cdot), a_{i}(t, \cdot) \in C^{N+1}\left(\mathbb{R}^{d}\right)$, with their partial derivatives of all orders bounded by $M$, uniformly with respect to $t \in\left[0, T_{0}\right]$.

Under Assumption 4.1 it is well-known that $\mathcal{A}(t)$ admits a fundamental solution $\Gamma(t, z ; T, \zeta)$, which is the solution of the Cauchy problem (2.2) with $\varphi=\delta_{\zeta}$. Equivalently, for any $\left.T \in\right] 0, T_{0}$ [ and for any measurable function $\varphi$ with at most exponential growth, the backward parabolic Cauchy problem (2.2) admits a unique classical solution $u$, which is given by

$$
u(t, z)=\int_{\mathbb{R}^{d}} \Gamma(t, z ; T, \zeta) \varphi(\zeta) \mathrm{d} \zeta, \quad t \in\left[0, T\left[, z \in \mathbb{R}^{d} .\right.\right.
$$


Furthermore, by the Feynman-Kac representation theorem, the function $\Gamma(t, z ; T, \zeta)$ is also the transition density of the stochastic process generated by $\mathcal{A}(t)$.

Remark 4.2. Assumption 4.1 can be considerably relaxed. The main results (Theorem 4.5 and Corollary 4.6 below) have been recently extended in Pagliarani and Pascucci (2014), to include the majority of popular models in mathematical finance (e.g. CEV, Heston, SABR, three-halves, etc.).

Consider now the Taylor polynomial expansion discussed in Example 2.3. It will be helpful to explicitly indicate the dependence on the expansion point $\bar{z}$. In particular, for any $\bar{z} \in \mathbb{R}^{d}$, we consider the polynomial expansion $\left(\mathcal{A}_{n}^{(\bar{z})}(t)\right)_{0 \leq n \leq N}$, given by

$$
\mathcal{A}_{n}^{(\bar{z})}(t, z) \equiv \mathcal{A}_{n}^{(\bar{z})}(t):=\sum_{|\alpha| \leq 2} a_{\alpha, n}^{(\bar{z})}(t, z) D_{z}^{\alpha} \quad a_{\alpha, n}^{(\bar{z})}(\cdot, z)=\sum_{|\beta|=n} \frac{D_{z}^{\beta} a_{\alpha}(\cdot, \bar{z})}{\beta !}(z-\bar{z})^{\beta}, \quad n \leq N
$$

Now, fix a maturity date $T$. We define the $N$-th order Taylor approximation centered at $\bar{z} \in \mathbb{R}^{d}$, of $\Gamma$ and $u$ respectively, as

$$
\bar{u}_{N}^{(\bar{z})}(t, z):=\sum_{n=0}^{N} u_{n}^{(\bar{z})}(t, z), \quad \bar{\Gamma}_{N}^{(\bar{z})}(t, z, T, \zeta):=\sum_{n=0}^{N} \Gamma_{n}^{(\bar{z})}(t, z, T, \zeta), \quad t<T, \quad z, \zeta \in \mathbb{R}^{d},
$$

where the functions

$$
u_{n}^{(\bar{z})}(t, \cdot)=\mathcal{L}_{n}^{(\bar{z})}(t, T) u_{0}^{(\bar{z})}(t, \cdot), \quad \quad \Gamma_{n}^{(\bar{z})}(t, \cdot ; T, \zeta)=\mathcal{L}_{n}^{(\bar{z})}(t, T) \Gamma_{0}^{(\bar{z})}(t, \cdot ; T, \zeta),
$$

are as given in Theorem 2.6. Note that $\bar{u}_{N}^{(\bar{z})}$ is defined for a fixed $T$, as indicated by (4.4). Note also that we have once again used the superscript $\bar{z}$ above to emphasize the dependence on the initial point of the Taylor expansion. For the particular choice $\bar{z}=z$, we give the following definition:

Definition 4.3. For a fixed maturity date $T$, we define the $N$-th order Taylor approximations of $u$ and $\Gamma$, respectively, as

$$
\bar{u}_{N}(t, z):=\bar{u}_{N}^{(z)}(t, z), \quad \bar{\Gamma}_{N}(t, z ; T, \zeta):=\bar{\Gamma}_{N}^{(z)}(t, z ; T, \zeta)
$$

where $\bar{u}_{N}^{(z)}(t, z)$ and $\bar{\Gamma}_{N}^{(z)}(t, z ; T, \zeta)$ are as defined in (4.3)-(4.4).

We now give analogous definitions for the implied volatility expansion. As we did in Section 3, we use the notation $(x, y) \in \mathbb{R} \times \mathbb{R}^{d-1}$ to indicate a point in $\mathbb{R}^{d}$, where we separate $x$ from all other components in order to distinguish the log-price from all the other variables (e.g. variance process, vol-vol process, etc.). For a Call option with maturity date $T$ and $\log$ strike $k$, we define the $N$-th order Taylor approximation centered at $(\bar{x}, \bar{y}) \in \mathbb{R} \times \mathbb{R}^{d-1}$ of the implied volatility $\sigma$, as

$$
\bar{\sigma}_{N}^{(\bar{x}, \bar{y})}(t, x, y, k):=\sigma_{0}^{(\bar{x}, \bar{y})}(t)+\sum_{n=0}^{N} \sigma_{n}^{(\bar{x}, \bar{y})}(t, x, y, k), \quad t<T, \quad(x, y) \in \mathbb{R} \times \mathbb{R}^{d-1},
$$

where, for sake of clarity we recall

$$
\begin{aligned}
\sigma_{0}^{(\bar{x}, \bar{y})}(t) & =\sqrt{\frac{2}{T-t} \int_{t}^{T} a_{(2,0, \ldots, 0)}(s, \bar{x}, \bar{y}) \mathrm{d} s} \\
\sigma_{n}^{(\bar{x}, \bar{y})}(t, x, y, k) & =\frac{u_{n}^{(\bar{x}, \bar{y})}(t, x, y, k)}{\partial_{\sigma} u^{\mathrm{BS}}\left(\sigma_{0}^{(\bar{x}, \bar{y})}(t) ; T-t, x, k\right)}-\frac{1}{n !} \sum_{h=2}^{n} \mathbf{B}_{n, h}\left(1 ! \sigma_{1}^{(\bar{x}, \bar{y})}, 2 ! \sigma_{2}^{(\bar{x}, \bar{y})}, \ldots,(n-h+1) ! \sigma_{n-h+1}^{(\bar{x}, \bar{y})}\right)
\end{aligned}
$$




$$
\begin{gathered}
\times \frac{\partial_{\sigma}^{h} u^{\mathrm{BS}}\left(\sigma_{0}^{(\bar{x}, \bar{y})}(t) ; T-t, x, k\right)}{\partial_{\sigma} u^{\mathrm{BS}}\left(\sigma_{0}^{(\bar{x}, \bar{y})}(t) ; T-t, x, k\right)}, \quad n \geq 1, \\
u_{n}^{(\bar{x}, \bar{y})}(t, x, y, k)=\mathcal{L}_{n}^{(\bar{x}, \bar{y})}(t, T) u_{0}^{(\bar{x}, \bar{y})}(t, x, k)=\mathcal{L}_{n}^{(\bar{x}, \bar{y})}(t, T) u^{\mathrm{BS}}\left(\sigma_{0}^{(\bar{x}, \bar{y})}(t) ; T-t, x, k\right) .
\end{gathered}
$$

A few notes are in order. First, we have added the $\operatorname{argument} k$ to the function $u_{n}^{(\bar{x}, \bar{y})}$ to indicate its dependence on $\log$ strike. Second, the function $u_{n}^{(\bar{x}, \bar{y})}$ depends on the maturity date T, as indicated by (4.9). Third, each $\sigma_{n}^{(\bar{x}, \bar{y})}$ in the sequence $\left(\sigma_{n}^{(\bar{x}, \bar{y})}\right)_{n \geq 1}$ depends on $(t, x, y, k)$. Though, for clarity, we have not written all of these arguments in $\mathbf{B}_{n, h}\left(1 ! \sigma_{1}^{(\bar{x}, \bar{y})}, 2 ! \sigma_{2}^{(\bar{x}, \bar{y})}, \ldots,(n-h+1) ! \sigma_{n-h+1}^{(\bar{x}, \bar{y})}\right)$. Fourth, we have once again explicitly indicated with a superscript $(\bar{x}, \bar{y})$ the dependence on the initial point of the Taylor expansion. For the particular choice $\bar{x}=x$ and $\bar{y}=y$, we make the following definition:

Definition 4.4. For a Call option with $\log$ strike $k$ and maturity $T$, we define the $N$-th order Taylor approximation of the implied volatility $\sigma$ as

$$
\bar{\sigma}_{N}(t, x, y, k):=\bar{\sigma}_{N}^{(x, y)}(t, x, y, k),
$$

where $\bar{\sigma}_{N}^{(x, y)}(t, x, y, k)$ is as defined in (4.6)-(4.7)-(4.8)-(4.9).

\subsection{Error estimates for the transition density and prices}

The following theorem provides an asymptotic pointwise estimate as $t \rightarrow T^{-}$for the error introduced by replacing the exact transition density $\Gamma$ with the $N$-th order approximation $\bar{\Gamma}_{N}$.

Theorem 4.5. Let Assumption 4.1 hold and let $0<T \leq T_{0}$. Then, for any $\varepsilon>0$ we have

$$
\left|\Gamma(t, z ; T, \zeta)-\bar{\Gamma}_{N}(t, z ; T, \zeta)\right| \leq C(T-t)^{\frac{N+1}{2}} \Gamma^{M+\varepsilon}(t, z ; T, \zeta), \quad 0 \leq t<T, z, \zeta \in \mathbb{R}^{d},
$$

where $\bar{\Gamma}_{N}(t, z ; T, \zeta)$ is as defined in (4.5), $\Gamma^{M+\varepsilon}(t, z ; T, \zeta)$ is the fundamental solution of the heat operator

$$
H^{M+\varepsilon}=(M+\varepsilon) \sum_{i=1}^{d} \partial_{z_{i}}^{2}+\partial_{t}
$$

and $C$ is a positive constant that depends only on $M, N, T_{0}$ and $\varepsilon$.

Combining Theorem 4.5 with (4.1) we obtain an asymptotic estimate for $\left|u(t, z)-\bar{u}_{N}(t, z)\right|$, the pricing error.

Corollary 4.6. Under the assumptions of Theorem 4.5, for any $0<T \leq T_{0}, \varepsilon>0$ we have

$$
\left|u(t, z)-\bar{u}_{N}(t, z)\right| \leq C(T-t)^{\frac{N+1}{2}} \int_{\mathbb{R}^{d}} \Gamma^{M+\varepsilon}(t, z ; T, \zeta) \varphi(\zeta) \mathrm{d} \zeta, \quad 0 \leq t<T, z \in \mathbb{R}^{d} .
$$

where $\bar{u}_{N}(t, z)$ is as defined in (4.5).

The proof of Theorem 4.5 relies on the following Gaussian estimates (see Friedman (1964), Chapter 1).

Lemma 4.7. Let $\mathcal{A}(t)$ be a differential operator satisfying Assumption 4.1 and let $\Gamma=\Gamma(t, z ; T, \zeta)$ be the fundamental solution corresponding to $\mathcal{A}(t)$. Then, for any $\varepsilon>0$ and $\beta, \gamma \in \mathbb{N}_{0}^{d}$ with $|\gamma| \leq N+3$, we have

$$
\left|(z-\zeta)^{\beta} D_{z}^{\gamma} \Gamma(t, z ; T, \zeta)\right| \leq C(T-t)^{\frac{|\beta|-|\gamma|}{2}} \Gamma^{M+\varepsilon}(t, z ; T, \zeta), \quad 0 \leq t<T \leq T_{0}, \quad z, \zeta \in \mathbb{R}^{d},
$$

where $\Gamma^{M+\varepsilon}$ is the fundamental solution of the heat operator (4.12) and $C$ is a positive constant, which depends only on $M, N, T_{0}, \varepsilon$ and $|\beta|$. 
We also need the following preliminary estimates (see (Lorig et al., 2013, Lemma 6.23))

Lemma 4.8. Under the assumptions of Theorem 4.5, for any $n \in \mathbb{N}$ with $n \leq N, \epsilon>0$, and for any $\beta \in \mathbb{N}_{0}^{d}$, we have

$$
\left|D_{z}^{\beta} \Gamma_{n}^{(\bar{z})}(t, z)\right| \leq C(T-t)^{\frac{n-|\beta|}{2}}\left(1+|z-\bar{z}|^{n}(T-t)^{-\frac{n}{2}}\right) \Gamma^{M+\varepsilon}(t, z ; T, \zeta),
$$

which holds for $0 \leq t<T \leq T_{0}, z, \zeta, \bar{z} \in \mathbb{R}^{d}$. Here, the function $\Gamma^{M+\varepsilon}$ is the fundamental solution of the heat operator (4.12) and $C$ is a positive constant, which depends only on $M, N, T_{0}, \varepsilon$ and $|\beta|$.

Proof of Theorem 4.5. From (Lorig et al., 2013, Theorem 3.8), for any given $T \leq T_{0}$, the functions $\left(u_{n}^{(\bar{z})}\right)_{n \geq 1}$ given by (2.17)-(2.18) can be equivalently defined as the unique non-rapidly increasing solutions of the following sequence of nested heat-type Cauchy problems:

$$
\begin{cases}\left(\partial_{t}+\mathcal{A}_{0}^{(\bar{z})}(t)\right) u_{n}^{(\bar{z})}(t, z)=-\sum_{h=1}^{n} \mathcal{A}_{h}^{(\bar{z})}(t) u_{n-h}^{(\bar{z})}(t, z), & t<T, z \in \mathbb{R}^{d}, \\ u_{n}^{(\bar{z})}(T, z)=0, & z \in \mathbb{R}^{d} .\end{cases}
$$

The thesis then follows directly from (Lorig et al., 2013, Theorem 3.10). For completeness, we provide here a sketch of the proof given in Lorig et al. (2013). By (4.16) it is easy to prove that $v^{(\bar{z})}:=u-\bar{u}_{N}^{(\bar{z})}$ solves

$$
\begin{cases}\left(\partial_{t}+\mathcal{A}(t)\right) v^{(\bar{z})}(t, z)=-\sum_{n=0}^{N}\left(\mathcal{A}(t)-\overline{\mathcal{A}}_{n}^{(\bar{z})}(t)\right) u_{N-n}^{(\bar{z})}(t, z), & t<T, z \in \mathbb{R}^{d}, \\ v^{(\bar{z})}(T, z)=0, & z \in \mathbb{R}^{d},\end{cases}
$$

where we have defined

$$
\overline{\mathcal{A}}_{n}^{(\bar{z})}(t)=\sum_{i=0}^{n} \mathcal{A}_{i}^{(\bar{z})}(t)
$$

Thus, by Duhamel's principle we obtain

$$
u(t, z)-\bar{u}_{N}(t, z)=\int_{t}^{T} \int_{\mathbb{R}^{d}} \Gamma(t, z ; s, \xi) \sum_{n=0}^{N}\left(\mathcal{A}(s)-\overline{\mathcal{A}}_{n}^{(z)}(s)\right) u_{N-n}^{(z)}(s, \xi) \mathrm{d} \xi \mathrm{d} s, \quad t<T, z \in \mathbb{R}^{d} .
$$

Now, by (4.2) we have

$$
\begin{aligned}
\left|\left(\mathcal{A}(s)-\overline{\mathcal{A}}_{n}^{(z)}(s)\right) u_{N-n}^{(z)}(s, \xi)\right| & \leq \sum_{|\alpha| \leq 2}\left|a_{\alpha}^{(z)}(s, \xi)-\sum_{i=0}^{n} a_{\alpha, n}^{(z)}(s, \xi)\right|\left|D_{\xi}^{\alpha} u_{N-n}^{(z)}(s, \xi)\right| \\
& =\sum_{|\alpha| \leq 2}\left|a_{\alpha}(s, \xi)-\sum_{i=0}^{n} \sum_{|\beta|=n} \frac{D_{z}^{\beta} a_{\alpha}(s, z)}{\beta !}(\xi-z)^{\beta}\right|\left|D_{\xi}^{\alpha} u_{N-n}^{(z)}(s, \xi)\right| \\
& \leq M|\xi-z|^{n+1} \sum_{|\alpha| \leq 2}\left|D_{\xi}^{\alpha} u_{N-n}^{(z)}(s, \xi)\right|,
\end{aligned}
$$

where the last line follows by the hypothesis (ii) in Assumption 4.1 on the coefficients $\left(a_{\alpha}\right)_{|\alpha| \leq 2}$. Finally, by considering $u_{n}^{(z)}(t, z)=\Gamma_{n}^{(z)}(t, z, ; T, \zeta)=\mathcal{L}_{n}^{(z)}(t, T) \Gamma_{0}^{(z)}(t, z ; T, \zeta)$, we obtain

$$
\left|\Gamma(t, z ; T, \zeta)-\bar{\Gamma}_{N}(t, z ; T, \zeta)\right| \leq M \sum_{n=0}^{N} \sum_{|\alpha| \leq 2} \int_{t}^{T} \int_{\mathbb{R}^{d}} \Gamma(t, z ; s, \xi)|\xi-z|^{n+1}\left|D_{\xi}^{\alpha} \Gamma_{N-n}^{(z)}(s, \xi ; T, \zeta)\right| \mathrm{d} \xi \mathrm{d} s .
$$


The thesis now follows by repeatedly applying the Gaussian estimates (4.14) and (4.15), along with the semigroup property

$$
\int_{\mathbb{R}^{d}} \Gamma^{M+\varepsilon}(t, z ; s, \xi) \Gamma^{M+\varepsilon}(s, \xi ; T, \zeta) \mathrm{d} \xi \mathrm{d} s=\Gamma^{M+\varepsilon}(t, z ; T, \zeta) \quad t<s<T, \quad z, \zeta \in \mathbb{R}^{d} .
$$

\subsection{Short-time asymptotics for the implied volatility}

We provide error estimates for the $N$-th order implied volatility approximation $\bar{\sigma}_{N}$, defined in (4.10), on the subset $|x-k| \leq \lambda \sqrt{T-t}$ where $\lambda$ is an arbitrary, but fixed, positive constant.

Theorem 4.9. Let Assumption 4.1 hold and let $\lambda>0$. Denote by $\sigma(t, x, y, k)$ the exact implied volatility of a Call option, with $\log$ strike $k$ and maturity $T$. That is, $\sigma(t, x, y, k)$ is the unique positive solution of $u^{\mathrm{BS}}(\sigma ; T-t, x, k)=u(t, x, y, k)$, where $u$ is the classical solution $\sigma$ of (2.2) with time $T$ terminal conditions $\varphi(x)=\left(\mathrm{e}^{x}-\mathrm{e}^{k}\right)^{+}$. Then the $N$-th order implied volatility approximation $\bar{\sigma}_{N}(t, x, y, k)$, defined in (4.10), satisfies

$$
\left|\sigma(t, x, y, k)-\bar{\sigma}_{N}(t, x, y, k)\right| \leq C(T-t)^{\frac{N+1}{2}}, \quad 0 \leq t<T \leq T_{0}, y \in \mathbb{R}^{d-1},|x-k| \leq \lambda \sqrt{T-t},
$$

where $C$ is a positive constant that depends only on $M, N, T_{0}$ and $\lambda$.

Remark 4.10. In the particular case $d=1$, the above result is consistent with (Bompis and Gobet, 2012, Theorem 22) where an implied volatility approximation for local volatility models has been derived. A direct computation shows that such an expansion is equivalent to our $\bar{\sigma}_{2}$. Although Theorem 4.9 holds true for any order $N \in \mathbb{N}_{0}$, and any dimension $d \in \mathbb{N}$, the estimate in Bompis and Gobet (2012) was proved by the authors under milder assumptions for the generator $\mathcal{A}(t)$, and for three different choices of the initial point $\bar{x}$ of the Taylor expansion: $\bar{x}=x, \bar{x}=k$ and $\bar{x}=\frac{x+k}{2}$.

Remark 4.11. Theorem 4.9 also provides us with an explicit representation for the $n$-th order derivative with respect to $T$, of the implied volatility surface at $x=k$ and $T=t$. More precisely, as a corollary of (4.17) we have:

$$
\left.\partial_{t}^{n} \sigma(t, x, y, k)\right|_{t=T, k=x}=\left.\partial_{t}^{n} \bar{\sigma}_{N}(t, x, y, k)\right|_{t=T, k=x}, \quad \forall N \geq 2 n .
$$

A direct computation shows that, for $n=0$, the representation (4.18) is consistent with the well-known results by Berestycki et al. (2002) and Berestycki et al. (2004). It is also easy to check that our expansion gives the correct slope of the implied volatility at the money in the limit as $t \rightarrow T$. For the special case $d=1$, we recover the practitioners' $1 / 2$ slope rule, which gives the at-the-money slope of implied volatility as one half the slope of the local volatility function.

In what follows, the maturity date $T \in\left(0, T_{0}\right]$ is fixed. We recall the Black-Scholes price

$$
u^{\mathrm{BS}}(\sigma)=u^{\mathrm{BS}}(\sigma ; T-t, x, k),
$$

as it is in Definition 3.1 and we denote by $\left(u^{\mathrm{BS}}\right)^{-1}(u ; T-t, x, k)=\left(u^{\mathrm{BS}}\right)^{-1}(u)$ its inverse with respect to the $\sigma$ variable. We also introduce the following function:

$$
u(\delta)=u(\delta ; t, x, y, k):=\sum_{n=0}^{N} \delta^{n} u_{n}^{(x, y)}(t, x, y, k)+\delta^{N+1}\left(u-\bar{u}_{N}\right)(t, x, y, k)
$$




$$
=u^{\mathrm{BS}}\left(\sigma_{0}^{(x, y)}(t) ; T-t, x, k\right)+\sum_{n=1}^{N} \delta^{n} u_{n}^{(x, y)}(t, x, y, k)+\delta^{N+1}\left(u-\bar{u}_{N}\right)(t, x, y, k), \quad \delta \in[0,1],
$$

where we have used Remark 3.2 Note that the function $u_{n}^{(x, y)}(t, x, y, k)$ is defined for a fixed maturity date $T$, as indicated by (4.9).

Remark 4.12. It is possible to prove (see Lorig et al. (2013)) that, in the case of a Call option, the estimate (4.13), as well as (4.15), can be improved by exploiting the local Lipschitz continuity of the payoff $\varphi(x)=\left(e^{x}-e^{k}\right)^{+}$. More precisely, it is possible to prove that, for any log-strike $k \in \mathbb{R}$, we have

$$
\left|u(t, x, y, k)-\bar{u}_{N}(t, x, y, k)\right| \leq C(T-t)^{\frac{N+2}{2}} u^{\mathrm{BS}}(\sqrt{2 M} ; T-t, x, k), \quad 0 \leq t<T, \quad(x, y) \in \mathbb{R} \times \mathbb{R}^{d-1},
$$

and that, for any $n \in \mathbb{N}$ with $n \leq N$, we also have

$$
\left|u_{n}^{(x, y)}(t, x, y, k)\right| \leq C(T-t)^{\frac{n+1}{2}} u^{\mathrm{BS}}(\sqrt{2 M} ; T-t, x, k), \quad 0 \leq t<T,(x, y) \in \mathbb{R} \times \mathbb{R}^{d-1}, k \in \mathbb{R},
$$

where, as in Theorem 4.9, $C$ is a positive constant that only depends on $M, N$ and $T_{0}$.

The proof of Theorem 4.9 is based on the previous remark and some asymptotic estimates of the BlackScholes price for short-maturities, which are proved in Appendix $\mathrm{A}$.

Lemma 4.13. Let $u(\delta)$ be as in (4.19). Under the assumptions of Theorem 4.9, there exists $\tau_{0}>0$, only dependent on $M, N, T_{0}$ and $\lambda$, such that

$$
u^{\mathrm{BS}}(\sqrt{2 / M} ; T-t, x, k) \leq u(\delta) \leq u^{\mathrm{BS}}(\sqrt{2 M} ; T-t, x, k),
$$

or equivalently

$$
\sqrt{2 / M} \leq\left(u^{\mathrm{BS}}\right)^{-1}(u(\delta) ; T-t, x, k) \leq \sqrt{2 M},
$$

for any $t \in\left[T-\tau_{0}, T\right),|x-k| \leq \lambda \sqrt{T-t}, y \in \mathbb{R}^{d-1}$ and $\delta \in[0,1]$.

Proof. Throughout this proof $C$ will always denote a positive constant that depends only on $M, N, T_{0}$ and $\lambda$. By Remark 4.12, and since $\delta \in[0,1]$, we obtain

$$
\left|\sum_{n=1}^{N} \delta^{n} u_{n}^{(x, y)}(t, x, y, k)+\delta^{N+1}\left(u-\bar{u}_{N}\right)(t, x, y, k)\right| \leq C(T-t) u^{\mathrm{BS}}(\sqrt{2 M} ; T-t, x, k)
$$

(using Lemma A.1 and Assumption 4.1)

$$
\leq C(T-t) u^{\mathrm{BS}}\left(\sigma_{0}^{(x, y)}(t) ; T-t, x, k\right)
$$

for any $0 \leq t<T, y \in \mathbb{R}^{d-1}$ and $|x-k| \leq \lambda \sqrt{T-t}$. Combining (4.19) and (4.21), we obtain

$$
u(\delta) \geq(1-C(T-t)) u^{\mathrm{BS}}\left(\sigma_{0}^{(x, y)}(t) ; T-t, x, k\right) .
$$

The lower bound for $u(\delta)$ in (4.20) now follows from inequality (A.7) in Lemma A.2. To establish the upper bound for $u(\delta)$, we combine (4.19) with (4.21) to obtain

$$
u(\delta) \leq(1+C(T-t)) u^{\mathrm{BS}}\left(\sigma_{0}^{(x, y)}(t) ; T-t, x, k\right) .
$$

The upper bound in (4.20) now follows from inequality (A.8) in Lemma A.2. 
Lemma 4.14. Under the assumptions of Theorem 4.9, for any $N \in \mathbb{N}$ there exist positive constants $C$ and $\tau_{0}$, only dependent on $M, N, T_{0}$ and $\lambda$, such that

$$
\left|\partial_{u}^{n}\left(u^{\mathrm{BS}}\right)^{-1}(u(\delta ; t, x, y, k) ; T-t, x, k)\right| \leq C\left(e^{k} \sqrt{T-t}\right)^{-n}
$$

for any $n \leq \mathbb{N}, t \in\left[T-\tau_{0}, T\right),|x-k| \leq \lambda \sqrt{T-t}, y \in \mathbb{R}^{d-1}$ and $\delta \in[0,1]$.

Proof. Throughout this proof, $C$ will always denote a positive constant only dependent on $M, N, T_{0}$ and $\lambda$. Note that, for any $\sigma>0$ we have

$$
\partial_{\sigma} u^{\mathrm{BS}}(\sigma) \equiv \partial_{\sigma} u^{\mathrm{BS}}(\sigma ; T-t, x, k)=\frac{e^{k} \sqrt{T-t}}{\sqrt{2 \pi}} \exp \left(-\frac{\left(\sigma^{2}(T-t)-2(x-k)\right)^{2}}{8 \sigma^{2}(T-t)}\right),
$$

and thus

$$
\frac{e^{k} \sqrt{T-t}}{\sqrt{2 \pi}} \exp \left(-\frac{\sigma^{2} T_{0}}{8}-\frac{\lambda^{2}}{2 \sigma^{2}}-\frac{\lambda \sqrt{T_{0}}}{2}\right) \leq \partial_{\sigma} u^{\mathrm{BS}}(\sigma) \leq \frac{e^{k} \sqrt{T-t}}{\sqrt{2 \pi}}, \quad 0 \leq t<T,|x-k| \leq \lambda \sqrt{T-t} .
$$

Therefore, by Lemma 4.13, there exists a positive $\tau_{0}$, only dependent on $M, N, T_{0}$ and $\lambda$, such that

$$
C \frac{e^{k} \sqrt{T-t}}{\sqrt{2 \pi}} \leq \partial_{\sigma} u^{\mathrm{BS}}\left(\left(u^{\mathrm{BS}}\right)^{-1}(u(\delta))\right) \leq \frac{e^{k} \sqrt{T-t}}{\sqrt{2 \pi}},
$$

for any $y \in \mathbb{R}^{d-1}, t \in\left[T-\tau_{0}, T\right),|x-k| \leq \lambda \sqrt{T-t}$ and $\delta \in[0,1]$, where $C$ is the positive constant

$$
C=\min _{\sigma \in[\sqrt{2 / M}, \sqrt{2 M}]} \exp \left(-\frac{\sigma^{2} T_{0}}{8}-\frac{\lambda^{2}}{2 \sigma^{2}}-\frac{\lambda \sqrt{T_{0}}}{2}\right) .
$$

Furthermore, by combining the second inequality in (4.23) with Proposition 3.5, we also obtain

$$
\left|\partial_{\sigma}^{n} u^{\mathrm{BS}}\left(\left(u^{\mathrm{BS}}\right)^{-1}(u(\delta))\right)\right| \leq C e^{k} \sqrt{T-t} .
$$

We are now prove the thesis by induction on $n$. The case $n=1$ clearly follows from the first inequality in (4.23). We have

$$
\left|\partial_{u}\left(u^{\mathrm{BS}}\right)^{-1}(u(\delta))\right|=\frac{1}{\partial_{\sigma} u^{\mathrm{BS}}\left(\left(u^{\mathrm{BS}}\right)^{-1}(u(\delta))\right)} \leq \frac{C e^{-k}}{\sqrt{T-t}} .
$$

Let us now assume (4.22) holds true for any $m \leq n$, and prove it holds true for $n+1$. By Faà di Bruno's formula (see Appendix B, Eq. (B.1)), we have

$$
\partial_{u}^{n+1}\left(u^{\mathrm{BS}}\right)^{-1}(u)=\frac{\sum_{h=2}^{n+1} \partial_{\sigma}^{h} u^{\mathrm{BS}}\left(\left(u^{\mathrm{BS}}\right)^{-1}(u)\right) \mathbf{B}_{n+1, h}\left(\partial_{u}\left(u^{\mathrm{BS}}\right)^{-1}(u), \cdots, \partial_{u}^{n-h+2}\left(u^{\mathrm{BS}}\right)^{-1}(u)\right)}{\partial_{\sigma} u^{\mathrm{BS}}\left(\left(u^{\mathrm{BS}}\right)^{-1}(u)\right)},
$$

and thus, by (4.23) and (4.24), we obtain

$$
\begin{aligned}
& \left|\partial_{u}^{n+1}\left(u^{\mathrm{BS}}\right)^{-1}(u(\delta))\right| \leq C \sum_{h=2}^{n+1}\left|\mathbf{B}_{n+1, h}\left(\partial_{u}\left(u^{\mathrm{BS}}\right)^{-1}(u(\delta)), \cdots, \partial_{u}^{n-h+2}\left(u^{\mathrm{BS}}\right)^{-1}(u(\delta))\right)\right| \\
& \leq C \sum_{h=2}^{n+1} \sum_{j_{1}, \cdots, j_{n-h+2}}\left|\partial_{u}\left(u^{\mathrm{BS}}\right)^{-1}(u(\delta))\right|^{j_{1}} \cdots\left|\partial_{u}^{n-h+2}\left(u^{\mathrm{BS}}\right)^{-1}(u(\delta))\right|^{j_{n-h+2}} \quad \text { (by (B.2) in Appendix (B) }
\end{aligned}
$$




$$
\begin{aligned}
& \leq C \sum_{h=2}^{n+1} \sum_{j_{1}, \cdots, j_{n-h+2}}\left(e^{k \sqrt{T-t}}\right)^{-j_{1}} \cdots\left(e^{k} \sqrt{T-t}\right)^{-j_{n-h+2}} \\
& \leq C \sum_{h=2}^{n+1} \sum_{j_{1}, \cdots, j_{n-h+2}}\left(e^{k \sqrt{T-t}}\right)^{-(n+1)}=C\left(e^{k} \sqrt{T-t}\right)^{-(n+1)},
\end{aligned}
$$

(by inductive hypothesis)

where the last inequality follows from the second identity of (B.3) in Appendix $B$. This concludes the proof.

Proof of Theorem 4.9. Throughout this proof $C$ will indicate a positive constant only dependent on $M, N, T_{0}$ and $\lambda$. It suffices to prove the thesis (4.17) for small $T-t$. We start by recalling the function $g(\delta)$, which has already been used in Section 3.1 to carry out the formal expansion of the implied volatility, i.e.

$$
g(\delta)=g(\delta ; t, x, y, k):=\left(u^{\mathrm{BS}}\right)^{-1}(u(\delta ; t, x, y, k) ; T-t, x, k), \quad \delta \in[0,1] .
$$

By definition of $u(\delta)$ in (4.19), it is clear that

$$
\sigma(t, x, y, k)=g(1 ; t, x, y, k)
$$

Furthermore, with $\bar{\sigma}_{N}(t, x, y, k)$ as defined in (4.10), we have

$$
\bar{\sigma}_{N}(t, x, y, k)=\sigma_{0}^{(x, y)}(t)+\sum_{n=0}^{N} \sigma_{n}^{(x, y)}(t, x, y, k)=\left.\sum_{n=0}^{N} \frac{1}{n !} \partial_{\delta}^{n} g(\delta ; t, x, y, k)\right|_{\delta=0},
$$

since, by (4.19) and (3.6) we have, respectively, $\left.g(\delta)\right|_{\delta=0}=\sigma_{0}^{(x, y)}$, and $\left.\partial_{\delta}^{n} g(\delta)\right|_{\delta=0}=\sigma_{n}^{(x, y)}$ for $1 \leq n \leq N$. Now, by (4.25)-(4.26), and by the Taylor theorem with Lagrange remainder, there exist $\bar{\delta} \in[0,1]$ such that

$$
\begin{aligned}
\sigma-\bar{\sigma}_{N} & =g(1)-\sum_{n=0}^{N} \frac{1}{n !} \partial_{\delta}^{n} g(0)=\frac{1}{(N+1) !} \partial_{\delta}^{N+1} g(\bar{\delta}) \\
& =\frac{1}{(N+1) !} \sum_{h=1}^{N+1} \partial_{u}^{h}\left(u^{\mathrm{BS}}\right)^{-1}(u(\bar{\delta})) \mathbf{B}_{N+1, h}\left(\partial_{\delta} u(\bar{\delta}), \partial_{\delta}^{2} u(\bar{\delta}), \cdots, \partial_{\delta}^{N-h+2} u(\bar{\delta})\right),
\end{aligned}
$$

by (B.1) in Appendix B. Now, by (4.19) and Remark 4.12, we obtain

$$
\left|\partial_{\delta}^{n} u(\bar{\delta})\right| \leq C\left(\sum_{h=n}^{N}\left|u_{n}^{(x, y)}\right|+\left|u-\bar{u}_{N}\right|\right) \leq C(T-t)^{\frac{n+1}{2}} u^{\mathrm{BS}}(\sqrt{2 M} ; T-t, x, k) .
$$

Therefore, for any $1 \leq h \leq N+1$, by (B.2) in Appendix B we have

$$
\begin{aligned}
\left|\mathbf{B}_{N+1, h}\left(\partial_{\delta} u(\bar{\delta}), \partial_{\delta}^{2} u(\bar{\delta}), \cdots, \partial_{\delta}^{N-h+2} u(\bar{\delta})\right)\right| & \leq C \sum_{j_{1}, \cdots, j_{N-h+2}}\left|\partial_{\delta} u(\bar{\delta})\right|^{j_{1}}\left|\partial_{\delta}^{2} u(\bar{\delta})\right|^{j_{2}} \cdots\left|\partial_{\delta}^{N-h+2} u(\bar{\delta})\right|^{j_{N-h+2}} \\
& \leq C(\sqrt{T-t})^{N+h+1}\left(u^{\mathrm{BS}}(\sqrt{2 M} ; T-t, x, k)\right)^{h} .
\end{aligned}
$$

where in the last inequality we have used (4.28) and both the identities from (B.3) in Appendix B, Combining (4.22) and (4.29) with (4.27), we obtain

$$
\left|\sigma-\bar{\sigma}_{N}\right| \leq C(T-t)^{\frac{N+1}{2}} \sum_{h=1}^{N+1}\left(e^{-k} u^{\mathrm{BS}}(\sqrt{2 M} ; T-t, x, k)\right)^{h} .
$$

The thesis finally follows since $e^{-k} u^{\mathrm{BS}}(\sqrt{2 M} ; T-t, x, k) \leq e^{\lambda \sqrt{T-t}}$ for $|x-k| \leq \lambda \sqrt{T-t}$. 


\section{$5 \quad$ Implied volatility examples}

In this section we use the results of Section 3 to compute approximate model-induced implied volatilities under four different model dynamics in which European option prices can be computed explicitly.

- Section 5.1 CEV local volatility model

- Section 5.2 Heston stochastic volatility model

- Section 5.3 3/2 stochastic volatility model

- Section 5.4. SABR local-stochastic volatility model

We note that all of the above models fail to satisfy the rigorous assumptions required in Theorems 4.5 and 4.9 to prove the error bounds (4.11). However, as mentioned in Remark 4.2 Theorem 4.5 and Corollary 4.6 have been recently extended in Pagliarani and Pascucci (2014), to include all of the examples presented here.

In three of the four examples that follow we use a Taylor series polynomial expansion of $\mathcal{A}(t)$ as in Example 2.3. In these three cases, approximate implied volatilities can be computed using the formulas given in Appendix [C] For the Heston model, we use the time-dependent Taylor expansion of $\mathcal{A}(t)$ as in Example 2.4. In all cases, Mathematica notebooks containing the implied volatility formulas are available free of charge on the authors' website.

\subsection{CEV local volatility model}

In the Constant Elasticity of Variance (CEV) local volatility model of Cox (1975), the dynamics of the underlying $S$ are given by

$$
\mathrm{d} S_{t}=\delta S_{t}^{\beta-1} S_{t} \mathrm{~d} W_{t}, \quad S_{0}=s>0 .
$$

The parameter $\beta$ controls the relationship between volatility and price. When $\beta<1$, volatility increases as $S \rightarrow 0^{+}$. This feature, referred to as the leverage effect, is commonly observed in equity markets. When $\beta<1$, one also observes a negative at-the-money skew in the model-induced implied volatility surface. Like the leverage effect, a negative at-the-money skew is commonly observed in equity options markets. The origin is attainable when $\beta<1$. In order to prevent the process $S$ from taking negative values, one typically specifies zero as an absorbing boundary. Hence, the state space of $S$ is $[0, \infty)$. In $\log$ notation $X:=\log S$, we have the following dynamics 1

$$
\mathrm{d} X_{t}=-\frac{1}{2} \delta^{2} \mathrm{e}^{2(\beta-1) X_{t}} \mathrm{~d} t+\delta \mathrm{e}^{(\beta-1) X_{t}} \mathrm{~d} W_{t}, \quad X_{0}=x:=\log s .
$$

The generator of $X$ is given by

$$
\mathcal{A}=\frac{1}{2} \delta^{2} \mathrm{e}^{2(\beta-1) x}\left(\partial_{x}^{2}-\partial_{x}\right)
$$

Thus, from (2.4) we identify

$$
a(x, y)=\frac{1}{2} \delta^{2} \mathrm{e}^{2(\beta-1) x}, \quad b(x, y)=0, \quad c(x, y)=0, \quad f(x, y)=0 .
$$

\footnotetext{
${ }^{1}$ Here we define $\log 0:=\lim _{x \searrow 0} \log x=-\infty$.
} 
We fix a time to maturity $t$ and log-strike $k$. Using the formulas from Appendix Clas well as the Mathematica notebook provided on the authors' website, we compute explicitly

$$
\begin{aligned}
\sigma_{0} & =\delta \mathrm{e}^{(\beta-1) x}, \\
\sigma_{1} & =\frac{1}{2}(\beta-1) \sigma_{0}(k-x), \\
\sigma_{2} & =\frac{t}{24}(\beta-1)^{2} \sigma_{0}^{3}-\frac{t^{2}}{96}(\beta-1)^{2} \sigma_{0}^{5}+\frac{1}{12}(\beta-1)^{2} \sigma_{0}(k-x)^{2}, \\
\sigma_{3} & =\frac{t}{16}(\beta-1)^{3} \sigma_{0}^{3}(k-x)+\frac{-5 t^{2}}{192}(\beta-1)^{3} \sigma_{0}^{5}(k-x)
\end{aligned}
$$

In the CEV setting the exact price of a Call option is derived in Cox (1975):

$$
\begin{aligned}
u(t, x) & =e^{x} Q\left(\kappa, 2+\frac{2}{2-\beta}, 2 \chi\right)-\mathrm{e}^{k}\left(1-Q\left(2 \chi, \frac{2}{2-\beta}, 2 \kappa\right)\right), \\
Q(w, v, \mu) & =\sum_{n=0}^{\infty}\left(\frac{(\mu / 2)^{n} \mathrm{e}^{-\mu / 2}}{n !} \frac{\Gamma(v / 2+n, w / 2)}{\Gamma(v / 2+n)}\right), \\
\chi & =\frac{2 \mathrm{e}^{(2-\beta) x}}{\delta^{2}(2-\beta)^{2} t}, \\
\kappa & =\frac{2 \mathrm{e}^{(2-\beta) k}}{\delta^{2}(2-\beta)^{2} t},
\end{aligned}
$$

where $\Gamma(a)$ and $\Gamma(a, b)$ denote the complete and incomplete Gamma functions respectively. Thus, the implied volatility $\sigma$ can be obtained numerically by solving (3.3). In Figure 1 we plot our third order implied volatility approximation $\bar{\sigma}_{3}$ and the numerically obtained implied volatility $\sigma$. For comparison, we also plot the implied volatility expansion of Hagan and Woodward (1999)

$$
\sigma^{\mathrm{HW}}=\frac{\delta}{f^{1-\beta}}\left(1+\frac{(1-\beta)(2+\beta)}{24}\left(\frac{e^{x}-e^{k}}{f}\right)^{2}+\frac{(1-\beta)^{2}}{24} \frac{\delta^{2} t}{f^{2(1-\beta)}}+\cdots\right), \quad f=\frac{1}{2}\left(\mathrm{e}^{x}+\mathrm{e}^{k}\right) .
$$

\section{$5.2 \quad$ Heston stochastic volatility model}

Perhaps the most well-known stochastic volatility model is that of Heston (1993). In the Heston model, the dynamics of the underlying $S$ are given by

$$
\begin{aligned}
\mathrm{d} S_{t} & =\sqrt{Z_{t}} S_{t} \mathrm{~d} W_{t}, & & S_{0}=s>0, \\
\mathrm{~d} Z_{t} & =\kappa\left(\theta-Z_{t}\right) \mathrm{d} t+\delta \sqrt{Z_{t}} \mathrm{~d} B_{t}, & & Z_{0}=z>0, \\
\mathrm{~d}\langle W, B\rangle_{t} & =\rho \mathrm{d} t . & &
\end{aligned}
$$

As pointed out in Andersen and Piterbarg (2007), one must set $\rho<0$ in order to prevent a moment explosion. In order to improve the efficacy of our approximation it is convenient to perform the following change of variable $\left(X_{t}, V_{t}\right):=\left(\log S, e^{\kappa t} Z_{t}\right)$. Changing from $Z$ to $V$ removes the geometric part of the drift (see also Bompis and Gobet (2012)). By Ito's formula we obtain

$$
\begin{array}{rlrl}
\mathrm{d} X_{t} & =-\frac{1}{2} e^{-\kappa t} V_{t} \mathrm{~d} t+\sqrt{e^{-\kappa t} V_{t}} \mathrm{~d} W_{t}, & X_{0}=x:=\log s, \\
\mathrm{~d} V_{t} & =\theta \kappa e^{\kappa t} \mathrm{~d} t+\delta \sqrt{e^{\kappa t} V_{t}} \mathrm{~d} B_{t}, & V_{0}=v:=z>0, \\
\mathrm{~d}\langle W, B\rangle_{t} & =\rho \mathrm{d} t . & &
\end{array}
$$


The generator of $(X, V)$ is given by

$$
\mathcal{A}(t)=\frac{1}{2} e^{-\kappa t} v\left(\partial_{x}^{2}-\partial_{x}\right)+\theta \kappa e^{\kappa t} \partial_{v}+\frac{1}{2} \delta^{2} \delta e^{\kappa t} v \partial_{v}^{2}+\delta \rho v \partial_{x} \partial_{v} .
$$

Thus, using (2.4), we identify

$$
a(x, v)=\frac{1}{2} e^{-\kappa t} v, \quad b(x, v)=\delta^{2} \delta e^{\kappa t} v, \quad c(x, v)=\delta \rho v, \quad f(x, v)=\theta \kappa e^{\kappa t} .
$$

We fix a time to maturity $t$, a log-strike $k$, and we consider the time-dependent Taylor series expansion of $\mathcal{A}(t)$ as described in Example 2.4 with $(\bar{x}(t), \bar{v}(t))=\left(X_{0}, \mathbb{E}\left[V_{t}\right]\right):=\left(x, \theta\left(e^{\kappa t}-1\right)\right)$. Using the Mathematica notebook provided on the authors' website, we compute explicitly

$$
\begin{aligned}
\sigma_{0}= & \sqrt{\frac{-\theta+\theta \kappa t+e^{-\kappa t}(\theta-v)+v}{\kappa t}}, \\
\sigma_{1}= & \frac{\delta \rho z e^{-\kappa t}\left(-2 \theta-\theta \kappa t-e^{\kappa t}(\theta(\kappa t-2)+v)+\kappa t v+v\right)}{\sqrt{2} \kappa^{2} \sigma_{0}^{2} t^{3 / 2}}, \\
\sigma_{2}= & \frac{\delta^{2} e^{-2 \kappa t}}{32 \kappa^{4} \sigma_{0}^{5} t^{3}}\left(-2 \sqrt{2} \kappa \sigma_{0}^{3} t^{3 / 2} z\left(-\theta-4 e^{\kappa t}(\theta+\kappa t(\theta-v))+e^{2 \kappa t}(\theta(5-2 \kappa t)-2 v)+2 v\right)\right. \\
& +\kappa \sigma_{0}^{2} t\left(4 z^{2}-2\right)\left(\theta+e^{2 \kappa t}\left(-5 \theta+2 \theta \kappa t+8 \rho^{2}(\theta(\kappa t-3)+v)+2 v\right)\right) \\
& +\kappa \sigma_{0}^{2} t\left(4 z^{2}-2\right)\left(4 e^{\kappa t}\left(\theta+\theta \kappa t+\rho^{2}(\theta(\kappa t(\kappa t+4)+6)-v(\kappa t(\kappa t+2)+2))-\kappa t v\right)-2 v\right) \\
& +4 \sqrt{2} \rho^{2} \sigma_{0} \sqrt{t} z\left(2 z^{2}-3\right)\left(-2 \theta-\theta \kappa t-e^{\kappa t}(\theta(\kappa t-2)+v)+\kappa t v+v\right)^{2} \\
& \left.+4 \rho^{2}\left(4\left(z^{2}-3\right) z^{2}+3\right)\left(-2 \theta-\theta \kappa t-e^{\kappa t}(\theta(\kappa t-2)+v)+\kappa t v+v\right)^{2}\right)-\frac{\sigma_{1}^{2}\left(4(x-k)^{2}-\sigma_{0}^{4} t^{2}\right)}{8 \sigma_{0}^{3} t} \\
z & =\frac{x-k-\frac{\sigma_{0}^{2} t}{2}}{\sigma_{0} \sqrt{2 t}} .
\end{aligned}
$$

The expression for $\sigma_{3}$ is too long to reasonably put in the text. However, the explicit form of $\sigma_{3}$ is provided in the Mathematica notebook on the authors' website.

The characteristic function of $X_{t}$ is computed explicitly in Heston (1993)

$$
\begin{aligned}
& \eta(t, x, y, \lambda):=\log \mathbb{E}_{x, y} \mathrm{e}^{\mathrm{i} \lambda X_{t}}=\mathrm{i} \lambda x+C(t, \lambda)+D(t, \lambda) \mathrm{e}^{y}, \\
& C(t, \lambda)=\frac{\kappa \theta}{\delta^{2}}\left((\kappa-\rho \delta \dot{\mathrm{i}} \lambda+d(\lambda)) t-2 \log \left[\frac{1-f(\lambda) \mathrm{e}^{d(\lambda) t}}{1-f(\lambda)}\right]\right), \\
& D(t, \lambda)=\frac{\kappa-\rho \delta \mathrm{i} \lambda+d(\lambda)}{\delta^{2}} \frac{1-\mathrm{e}^{d(\lambda) t}}{1-f(\lambda) \mathrm{e}^{d(\lambda) t}}, \\
& f(\lambda)=\frac{\kappa-\rho \delta \mathrm{i} \lambda+d(\lambda)}{\kappa-\rho \delta \mathrm{i} \lambda-d(\lambda)}, \\
& d(\lambda)=\sqrt{\delta^{2}\left(\lambda^{2}+\mathbf{i} \lambda\right)+(\kappa-\rho \mathbf{i} \lambda \delta)^{2}} .
\end{aligned}
$$

Thus, the price of a European Call option can be computed using standard Fourier methods

$$
u(t, x, y)=\frac{1}{2 \pi} \int_{\mathbb{R}} \mathrm{d} \lambda_{r} \mathrm{e}^{\eta(t, x, y, \lambda)} \widehat{\varphi}(\lambda), \quad \widehat{\varphi}(\lambda)=\frac{-\mathrm{e}^{k-\mathrm{i} k \lambda}}{\mathrm{i} \lambda+\lambda^{2}}, \quad \lambda=\lambda_{r}+\mathrm{i} \lambda_{i}, \quad \lambda_{i}<-1 .
$$

Note, since the Call option payoff $\varphi(x)=\left(\mathrm{e}^{x}-\mathrm{e}^{k}\right)^{+}$is not in $L^{1}(\mathbb{R})$, its Fourier transform $\widehat{\varphi}(\lambda)$ must be computed in a generalized sense by fixing an imaginary component of the Fourier variable $\lambda_{i}<-1$. Using 
(5.7) the implied volatility $\sigma$ can be computed to solving (3.3) numerically. In Figure 2 we plot our third order implied volatility approximation $\bar{\sigma}_{3}$ and the numerically obtained implied volatility $\sigma$. For comparison, we also plot the small-time near-the-money implied volatility expansion of Forde et al. (2012) (see Theorem 3.2 and Corollary 4.3)

$$
\begin{aligned}
\sigma^{\mathrm{FJL}}= & \left(g_{0}^{2}+g_{1} t+o(t)\right)^{1 / 2}, \\
g_{0}= & \mathrm{e}^{y / 2}\left(1+\frac{1}{4} \rho \delta(k-x) \mathrm{e}^{-y}+\frac{1}{24}\left(1-\frac{5 \rho^{2}}{2}\right) \delta^{2}(k-x)^{2} \mathrm{e}^{-2 y}\right)+\mathcal{O}\left((k-x)^{3}\right), \\
g_{1}=- & \frac{\delta^{2}}{12}\left(1-\frac{\rho^{2}}{4}\right)+\frac{\mathrm{e}^{y} \rho \delta}{4}+\frac{\kappa}{2}\left(\theta-\mathrm{e}^{y}\right)+\frac{1}{24} \rho \delta \mathrm{e}^{-y}\left(\delta^{2} \bar{\rho}^{2}-2 \kappa\left(\theta+\mathrm{e}^{y}\right)+\rho \delta \mathrm{e}^{y}\right)(k-x) \\
& +\frac{\delta^{2} \mathrm{e}^{-2 y}}{7680}\left(176 \delta^{2}-480 \kappa \theta-712 \rho^{2} \delta^{2}+521 \rho^{4} \delta^{2}+40 \rho^{3} \delta \mathrm{e}^{y}+1040 \kappa \theta \rho^{2}-80 \kappa \rho^{2} \mathrm{e}^{y}\right)(k-x)^{2} \\
& +\mathcal{O}\left((k-x)^{3}\right), \quad \quad \bar{\rho}=\sqrt{1-\rho^{2}} .
\end{aligned}
$$

\section{$5.3 \quad 3 / 2$ stochastic volatility model}

We consider now the $3 / 2$ stochastic volatility model. The risk-neutral dynamics of the underlying $S$ in this setting are given by

$$
\begin{aligned}
\mathrm{d} S_{t} & =\sqrt{Z_{t}} S_{t} \mathrm{~d} W_{t}, & & S_{0}=s>0, \\
\mathrm{~d} Z_{t} & =Z_{t}\left(\kappa\left(\theta-Z_{t}\right) \mathrm{d} t+\delta \sqrt{Z_{t}} \mathrm{~d} B_{t}\right), & & Z_{0}=z>0, \\
\mathrm{~d}\langle W, B\rangle_{t} & =\rho \mathrm{d} t . & &
\end{aligned}
$$

As in all stochastic volatility models, one typically sets $\rho<0$ in order to capture the leverage effect. The $3 / 2$ model is noteworthy in that it does not fall into the affine class of Duffie et al. (2000), and yet it still allows for European option prices to be computed in semi-closed form (as a Fourier integral). Notice however that the characteristic function (given in (5.11) below) involves special functions such as the Gamma and the confluent hypergeometric functions. Therefore, Fourier pricing methods are not an efficient means of computed prices. The importance of the $3 / 2$ model in the pricing of options on realized variance is well documented by Drimus (2012). In particular, the $3 / 2$ model allows for upward-sloping implied volatility of variance smiles while Heston's model leads to downward-sloping volatility of variance smiles, in disagreement with observed skews in variance markets.

In $\log$ notation $(X, Y):=(\log S, \log Z)$ we have the following dynamics

$$
\begin{array}{rlrl}
\mathrm{d} X_{t} & =-\frac{1}{2} \mathrm{e}^{Y_{t}} \mathrm{~d} t+\mathrm{e}^{\frac{1}{2} Y_{t}} \mathrm{~d} W_{t}, & X_{0}=x:=\log s, \\
\mathrm{~d} Y_{t} & =\left(\kappa\left(\theta-\mathrm{e}^{Y_{t}}\right)-\frac{1}{2} \delta^{2} \mathrm{e}^{Y_{t}}\right) \mathrm{d} t+\delta \mathrm{e}^{\frac{1}{2} Y_{t}} \mathrm{~d} B_{t}, & Y_{0}=y:=\log z, \\
\mathrm{~d}\langle W, B\rangle_{t} & =\rho \mathrm{d} t . & &
\end{array}
$$

The generator of $(X, Y)$ is given by

$$
\mathcal{A}=\frac{1}{2} \mathrm{e}^{y}\left(\partial_{x}^{2}-\partial_{x}\right)+\left(\kappa\left(\theta-\mathrm{e}^{y}\right)-\frac{1}{2} \delta^{2} \mathrm{e}^{y}\right) \partial_{y}+\frac{1}{2} \delta^{2} \mathrm{e}^{y} \partial_{y}^{2}+\rho \delta \mathrm{e}^{y} \partial_{x} \partial_{y}
$$

Thus, using (2.4), we identify

$$
a(x, y)=\frac{1}{2} \mathrm{e}^{y}, \quad b(x, y)=\frac{1}{2} \delta^{2} \mathrm{e}^{y}, \quad c(x, y)=\rho \delta \mathrm{e}^{y}, \quad f(x, y)=\kappa\left(\theta-\mathrm{e}^{y}\right)-\frac{1}{2} \delta^{2} \mathrm{e}^{y} .
$$


We fix a time to maturity $t$ and log-strike $k$. Using the formulas from Appendix Clas well as the Mathematica notebook provided on the authors' website, we compute explicitly

$$
\begin{aligned}
\sigma_{0}= & \mathrm{e}^{y / 2} \\
\sigma_{1}= & \frac{t}{8}\left(2 \theta \kappa \sigma_{0}-\sigma_{0}^{3}\left(\delta^{2}-\delta \rho+2 \kappa\right)\right)+\frac{1}{4} \delta \rho \sigma_{0}(k-x) \\
\sigma_{2}=\frac{t}{96} \delta^{2}\left(8-7 \rho^{2}\right) \sigma_{0}^{3} & \\
& +\frac{t^{2}}{384}\left(-36 \theta \kappa \sigma_{0}^{3}\left(\delta^{2}-\delta \rho+2 \kappa\right)+\sigma_{0}^{5}\left(13 \delta^{4}-26 \delta^{3} \rho+4 \delta^{2}\left(13 \kappa+4 \rho^{2}-1\right)-52 \delta \kappa \rho+52 \kappa^{2}\right)+20 \theta^{2} \kappa^{2} \sigma_{0}\right) \\
& +\frac{t}{96} \delta \rho \sigma_{0}\left(6 \theta \kappa-7 \sigma_{0}^{2}\left(\delta^{2}-\delta \rho+2 \kappa\right)\right)(k-x)-\frac{1}{48} \delta^{2}\left(\rho^{2}-2\right) \sigma_{0}(k-x)^{2} \\
\sigma_{3}=\frac{t^{2}}{256} \delta^{2} \sigma_{0}^{3}\left(5\left(3 \rho^{2}-4\right) \sigma_{0}^{2}\left(\delta^{2}-\delta \rho+2 \kappa\right)+2 \theta \kappa\left(8-7 \rho^{2}\right)\right) & \\
& +\frac{t^{3}}{3072}\left(-132 \theta^{2} \kappa^{2} \sigma_{0}^{3}\left(\delta^{2}-\delta \rho+2 \kappa\right)+10 \theta \kappa \sigma_{0}^{5}\left(13 \delta^{4}-26 \delta^{3} \rho+4 \delta^{2}\left(13 \kappa+4 \rho^{2}-1\right)-52 \delta \kappa \rho+52 \kappa^{2}\right)\right. \\
& \quad+\frac{t}{128} \delta^{3} \rho\left(4-3 \rho^{2}\right) \sigma_{0}^{3}(k-x)+\frac{t^{2} \delta \rho \sigma_{0}}{1536}\left(-84 \theta \kappa \sigma_{0}^{2}\left(\delta^{2}-\delta \rho+2 \kappa\right)\right)(k-x) \\
& +\frac{t^{2} \delta \rho \sigma_{0}}{1536}\left(+\sigma_{0}^{4}\left(45 \delta^{4}-90 \delta^{3} \rho+4 \delta^{2}\left(45 \kappa+14 \rho^{2}-4\right)-180 \delta \kappa \rho+180 \kappa^{2}\right)+20 \theta^{2} \kappa^{2}\right)(k-x) \\
& +\frac{t}{384} \delta^{2} \sigma_{0}\left(\left(\rho^{2}-8\right) \sigma_{0}^{2}\left(\delta^{2}-\delta \rho+2 \kappa\right)-2 \theta \kappa\left(\rho^{2}-2\right)\right)(k-x)^{2},
\end{aligned}
$$

To the best of our knowledge, the above formula is the first explicit implied volatility expansion for the $3 / 2$ model. The characteristic function of $X_{t}$ is given, for example, in Proposition 3.2 of Baldeaux and Badran (2012). We have

$$
\begin{array}{rlrl}
\mathbb{E}_{x, y} \mathrm{e}^{\mathrm{i} \lambda X_{t}} & =\mathrm{e}^{\mathrm{i} \lambda x} \frac{\Gamma(\gamma-f)}{\Gamma(\gamma)}\left(\frac{2}{\delta^{2} z}\right)^{f} M\left(f, \gamma, \frac{-2}{\delta^{2} z}\right), & z=\frac{\mathrm{e}^{y}}{\kappa \theta}\left(\mathrm{e}^{\kappa \theta t}-1\right), & \gamma=2\left(f+1-\frac{p}{\delta^{2}}\right)(5.1) \\
f & =-\left(\frac{1}{2}-\frac{p}{\delta^{2}}\right)+\left(\left(\frac{1}{2}-\frac{p}{\delta^{2}}\right)^{2}+2 \frac{q}{\delta^{2}}\right)^{1 / 2}, \quad p=-\kappa+\mathrm{i} \delta \rho \lambda, \quad q=\frac{1}{2}\left(\mathrm{i} \lambda+\lambda^{2}\right),
\end{array}
$$

where $\Gamma$ is a Gamma function and $M$ is a confluent hypergeometric function. Thus, the price of a European Call option can be computed using standard Fourier methods

$$
u(t, x, y)=\frac{1}{2 \pi} \int_{\mathbb{R}} \mathrm{d} \lambda_{r} \widehat{\varphi}(\lambda) \mathbb{E}_{x, y} \mathrm{e}^{\mathrm{i} \lambda X_{t}}, \quad \lambda=\lambda_{r}+\mathrm{i} \lambda_{i}, \quad \lambda_{i}<-1,
$$

where $\widehat{\varphi}(\lambda)$ is given in (5.7). Using (5.12) the implied volatility $\sigma$ can be computed to solving (3.3) numerically. In Figure 3 we plot our third order implied volatility approximation $\bar{\sigma}_{3}$ and the numerically obtained implied volatility $\sigma$.

\subsection{SABR local-stochastic volatility}

The SABR model of Hagan et al. (2002) is a local-stochastic volatility model in which the risk-neutral dynamics of $S$ are given by

$$
\mathrm{d} S_{t}=Z_{t} S_{t}^{\beta} \mathrm{d} W_{t}, \quad S_{0}=s>0,
$$




$$
\begin{aligned}
\mathrm{d} Z_{t} & =\delta Z_{t} \mathrm{~d} B_{t}, \quad Z_{0}=z>0, \\
\mathrm{~d}\langle W, B\rangle_{t} & =\rho \mathrm{d} t .
\end{aligned}
$$

Modeling $Z$ as a geometric Brownian motion results in a true implied volatility smile (i.e., upward sloping implied volatility for high strikes); this is in contrast to the CEV model, for which the model-induced implied volatility is monotone decreasing (for $\beta<1$ ). In $\log$ notation $(X, Y):=(\log S, \log Z$ ) we have, we have the following dynamics:

$$
\begin{array}{rlrl}
\mathrm{d} X_{t} & =-\frac{1}{2} \mathrm{e}^{2 Y_{t}+2(\beta-1) X_{t}} \mathrm{~d} t+\mathrm{e}^{Y_{t}+(\beta-1) X_{t}} \mathrm{~d} W_{t}, & X_{0}=x:=\log s, \\
\mathrm{~d} Y_{t} & =-\frac{1}{2} \delta^{2} \mathrm{~d} t+\delta \mathrm{d} B_{t}, & Y_{0}=y:=\log z, \\
\mathrm{~d}\langle W, B\rangle_{t} & =\rho \mathrm{d} t . & &
\end{array}
$$

The generator of $(X, Y)$ is given by

$$
\mathcal{A}=\frac{1}{2} \mathrm{e}^{2 y+2(\beta-1) x}\left(\partial_{x}^{2}-\partial_{x}\right)-\frac{1}{2} \delta^{2} \partial_{y}+\frac{1}{2} \delta^{2} \partial_{y}^{2}+\rho \delta \mathrm{e}^{y+(\beta-1) x} \partial_{x} \partial_{y} .
$$

Thus, using (2.4), we identify

$$
a(x, y)=\frac{1}{2} \mathrm{e}^{2 y+2(\beta-1) x}, \quad b(x, y)=\frac{1}{2} \delta^{2}, \quad c(x, y)=\rho \delta \mathrm{e}^{y+(\beta-1) x}, \quad f(x, y)=-\frac{1}{2} \delta^{2} .
$$

We fix a time to maturity $t$ and log-strike $k$. Using the formulas from Appendix Cas well as the Mathematica notebook provided on the authors' website, we compute explicitly

$$
\sigma_{0}=\mathrm{e}^{y+(\beta-1) x}, \quad \sigma_{1}=\sigma_{1,0}+\sigma_{0,1}, \quad \sigma_{2}=\sigma_{2,0}+\sigma_{1,1}+\sigma_{0,2}, \quad \sigma_{3}=\sigma_{3,0}+\sigma_{2,1}+\sigma_{1,2}+\sigma_{0,3}(5.14)
$$

where

$$
\begin{aligned}
\sigma_{1,0}= & \frac{1}{2}(k-x)(-1+\beta) \sigma_{0}, \\
\sigma_{0,1}= & \frac{1}{4} \delta\left(2(k-x) \rho+t \sigma_{0}\left(-\delta+\rho \sigma_{0}\right)\right) \\
\sigma_{2,0}= & \frac{t}{24}(\beta-1)^{2} \sigma_{0}^{3}-\frac{t^{2}}{96}(\beta-1)^{2} \sigma_{0}^{5}+\frac{1}{12}(\beta-1)^{2} \sigma_{0}(k-x)^{2}, \\
\sigma_{1,1}= & \frac{t}{12}(\beta-1) \delta \rho \sigma_{0}^{2}-\frac{t^{2}}{48}(\beta-1) \delta \rho \sigma_{0}^{4}+\frac{t}{24}(\beta-1) \delta \sigma_{0}\left(\delta+\rho \sigma_{0}\right)(k-x)-\frac{1}{3}(\beta-1) \delta \rho(k-x)^{2}, \\
\sigma_{0,2}= & \frac{t}{24} \delta^{2}\left(8-3 \rho^{2}\right) \sigma_{0}+\frac{t^{2}}{96} \delta^{2} \sigma_{0}\left(5 \delta^{2}+2 \sigma_{0}\left(\left(6 \rho^{2}-2\right) \sigma_{0}-7 \delta \rho\right)\right) \\
& \quad-\frac{t}{24} \delta^{2} \rho\left(\delta-3 \rho \sigma_{0}\right)(k-x)+\frac{\delta^{2}\left(2-3 \rho^{2}\right)}{12 \sigma_{0}}(k-x)^{2}, \\
\sigma_{3,0}= & \frac{t}{16}(\beta-1)^{3} \sigma_{0}^{3}(k-x)-\frac{-5 t^{2}}{192}(\beta-1)^{3} \sigma_{0}^{5}(k-x), \\
\sigma_{2,1}= & \frac{t^{2}}{288}(\beta-1)^{2} \delta \sigma_{0}^{3}\left(17 \rho \sigma_{0}-11 \delta\right)+\frac{t^{3}}{384}(\beta-1)^{2} \delta \sigma_{0}^{5}\left(3 \delta-5 \rho \sigma_{0}\right)+\frac{t}{16}(\beta-1)^{2} \delta \rho \sigma_{0}^{2}(k-x) \\
& +\frac{-3 t^{2}}{64}(\beta-1)^{2} \delta \rho \sigma_{0}^{4}(k-x)+\frac{t}{48}(\beta-1)^{2} \delta \sigma_{0}\left(\rho \sigma_{0}-2 \delta\right)(k-x)^{2}+\frac{5}{24}(\beta-1)^{2} \delta \rho(k-x)^{3}, \\
& \quad \frac{t^{2}}{72}(\beta-1) \delta^{2} \rho \sigma_{0}^{2}\left(\delta-7 \rho \sigma_{0}\right)+\frac{t^{3}}{96}(\beta-1) \delta^{2} \rho \sigma_{0}^{4}\left(2 \delta-3 \rho \sigma_{0}\right) \\
& +\frac{t}{144}(\beta-1) \delta^{2}\left(2-17 \rho^{2}\right) \sigma_{0}(k-x)+\frac{t^{2}}{192}(\beta-1) \delta^{2} \sigma_{0}\left(\delta^{2}-6 \delta \rho \sigma_{0}+2\left(\rho^{2}-1\right) \sigma_{0}^{2}\right)(k-x)
\end{aligned}
$$




$$
\begin{aligned}
& +\frac{t}{48}(\beta-1) \delta^{2} \rho\left(5 \rho \sigma_{0}-7 \delta\right)(k-x)^{2}+\frac{(\beta-1) \delta^{2}\left(16 \rho^{2}-7\right)}{24 \sigma_{0}}(k-x)^{3}, \\
\sigma_{0,3}= & \frac{t^{2}}{96} \delta^{3} \sigma_{0}\left(3 \delta\left(\rho^{2}-4\right)+\rho\left(26-9 \rho^{2}\right) \sigma_{0}\right) \\
& +\frac{t^{3}}{384} \delta^{3} \sigma_{0}\left(\sigma_{0}\left(19 \delta^{2} \rho+2 \sigma_{0}\left(\delta\left(8-21 \rho^{2}\right)+\rho\left(15 \rho^{2}-11\right) \sigma_{0}\right)\right)-3 \delta^{3}\right) \\
& +\frac{t}{48} \delta^{3} \rho\left(3 \rho^{2}-2\right)(k-x)-\frac{t^{2}}{192} \delta^{3} \rho\left(\delta^{2}+6 \sigma_{0}\left(\delta \rho+\left(1-2 \rho^{2}\right) \sigma_{0}\right)\right)(k-x) \\
& -\frac{t}{16} \delta^{3} \rho\left(\rho^{2}-1\right)(k-x)^{2}+\frac{\delta^{3} \rho\left(6 \rho^{2}-5\right)}{24 \sigma_{0}^{2}}(k-x)^{3},
\end{aligned}
$$

There is no formula for European option prices in the general SABR setting. However, for the special zero-correlation case $\rho=0$ the exact price of a European Call is computed in Antonov and Spector (2012):

$$
\begin{aligned}
u(t, x)= & \mathrm{e}^{(x+k) / 2} \frac{\mathrm{e}^{-\delta^{2} t / 8}}{\sqrt{2 \pi \delta^{2} t}}\left\{\frac{1}{\pi} \int_{0}^{\infty} \mathrm{d} V \int_{0}^{\pi} \mathrm{d} \phi \frac{1}{V}\left(\frac{V}{V_{0}}\right)^{-1 / 2} \frac{\sin \phi \sin (|\nu| \phi)}{b-\cos \phi} \exp \left(\frac{\xi_{\phi}^{2}}{2 \delta^{2} t}\right)\right. \\
& \left.\quad+\frac{\sin (|\nu| \pi)}{\pi} \int_{0}^{\infty} \mathrm{d} V \int_{0}^{\infty} \mathrm{d} \psi \frac{1}{V}\left(\frac{V}{V_{0}}\right)^{-1 / 2} \frac{\sinh \psi}{b-\cosh \psi} \mathrm{e}^{-|\nu| \psi} \exp \left(\frac{\xi_{\psi}^{2}}{2 \delta^{2} t}\right)\right\}+\left(\mathrm{e}^{x}-\mathrm{e}^{k}\right)^{+}, \\
\xi_{\phi}= & \arccos \left(\frac{q_{h}^{2}+q_{x}^{2}+V^{2}+V_{0}^{2}}{2 V V_{0}}-\frac{q_{h} q_{x}}{V V_{0}} \cos \phi\right), \\
\xi_{\psi}= & \arccos \left(\frac{q_{h}^{2}+q_{x}^{2}+V^{2}+V_{0}^{2}}{2 V V_{0}}+\frac{q_{h} q_{x}}{V V_{0}} \cosh \psi\right), \quad q_{x}=\frac{\mathrm{e}^{(1-\beta) x}}{1-\beta}, \quad \nu=\frac{-1}{2(1-\beta)}, \quad V_{0}=\frac{\mathrm{e}^{y}}{\delta} . \\
b= & \frac{q_{h}^{2}+q_{x}^{2}}{2 q_{h} q_{x}}, \quad q_{h}=\frac{\mathrm{e}^{(1-\beta) k}}{1-\beta}, \quad q^{2}, \quad
\end{aligned}
$$

Thus, in the zero-correlation setting, the implied volatility $\sigma$ can be obtained by using the above formula and then by solving (3.3) numerically. In Figure 4 we plot our third order implied volatility approximation $\bar{\sigma}_{3}$ and the numerically obtained implied volatility $\sigma$. For comparison, we also plot the implied volatility expansion of Hagan et al. (2002)

$$
\begin{aligned}
\sigma^{\mathrm{HKLW}} & =\delta \frac{x-k}{D(\zeta)}\left\{1+t \delta^{2}\left[\frac{2 \gamma_{2}-\gamma_{1}^{2}+1 / f^{2}}{24}\left(\frac{\mathrm{e}^{y+\beta f}}{\delta}\right)^{2}+\frac{\rho \gamma_{1} \mathrm{e}^{y+\beta f}}{4 \delta}+\frac{2-3 \rho^{2}}{24}\right]\right\}, \\
f & =\frac{1}{2}\left(\mathrm{e}^{x}+\mathrm{e}^{k}\right), \\
\zeta & =\frac{\delta \mathrm{e}^{-y}}{\beta-1}\left(\mathrm{e}^{(1-\beta) k}-\mathrm{e}^{(1-\beta) x}\right), \\
\gamma_{1} & =\beta / f \\
\gamma_{2} & =\beta(\beta-1) / f^{2} \\
D(\zeta) & =\log \left(\frac{\sqrt{1-2 \rho \zeta+\zeta^{2}}+\zeta-\rho}{1-\rho}\right) .
\end{aligned}
$$

Note that we use the "corrected" SABR formula, which appears in Obloj (2008).

\section{Conclusions and future work}

In this paper we consider a general class of parametric local-stochastic volatility models. In this setting, we provide a family of approximations - one for each polynomial expansion of $\mathcal{A}(t)$ - for (i) European-style 
option prices and (ii) implied volatilities. The terms in our option price expansions are expressed as a differential operator acting on the Black-Scholes price. Thus, to compute approximate prices, one requires only a normal CDF. Our implied volatility expansions are explicit, requiring no special functions nor any numerical integration. Thus, approximate implied volatilities can be computed even faster than option prices.

We carry out extensive computations using the Taylor series expansion of $\mathcal{A}(t)$. In particular, we establish the rigorous error bounds of our pricing and implied volatility approximations. We also implement our implied volatility expansion under four separate model dynamics: CEV local volatility, Heston stochastic volatility, 3/2 stochastic volatility, and SABR local-stochastic volatility. In each setting we demonstrate that our implied volatility expansion provides an excellent approximation of the true implied volatility over a large range of strikes and maturities.

\section{Thanks}

The authors would like to thank Mike Staunton and two anonymous referees for their thorough reading of this manuscript. Their suggestions have improved both the mathematical quality and readability of our results.

\section{A Asymptotics of the Black-Scholes price for short maturities}

We prove some results concerning the short-maturity behavior of the Black-Scholes price. Throughout this appendix $\tau$ denotes the time to maturity. We recall the following alternative expression for the Black-Scholes price, taken from Roper and Rutkowski (2009)

$$
u^{\mathrm{BS}}(\sigma ; \tau, x, k)=\left(e^{x}-e^{k}\right)^{+}+e^{x} \sqrt{\frac{\tau}{2 \pi}} \int_{0}^{\sigma} e^{-\frac{1}{2}\left(\frac{x-k}{w \sqrt{\tau}}+\frac{w \sqrt{\tau}}{2}\right)^{2}} d w .
$$

Now we set

$$
F\left(\sigma_{1}, \sigma_{2}, \tau, \lambda\right):=\int_{\sigma_{1}}^{\sigma_{2}} e^{-\frac{1}{2}\left(\frac{\lambda^{2}}{w^{2}}+\tau \frac{w^{2}}{4}\right)} d w, \quad \sigma_{1} \leq \sigma_{2} .
$$

and observe that, if

$$
|x-k| \leq \lambda \sqrt{\tau}
$$

for some $\lambda>0$, then we have

$$
\begin{aligned}
e^{-\frac{\lambda \sqrt{\tau}}{2}} F\left(\sigma_{1}, \sigma_{2}, \tau, \lambda\right) & =\int_{\sigma_{1}}^{\sigma_{2}} e^{-\frac{1}{2}\left(\frac{\lambda}{w}+\frac{w \sqrt{\tau}}{2}\right)^{2}} d w \leq \int_{\sigma_{1}}^{\sigma_{2}} e^{-\frac{1}{2}\left(\frac{x-k}{w \sqrt{\tau}}+\frac{w \sqrt{\tau}}{2}\right)^{2}} d w \\
& \leq \int_{\sigma_{1}}^{\sigma_{2}} e^{-\frac{1}{2}\left(\frac{\lambda}{w}-\frac{w \sqrt{\tau}}{2}\right)^{2}} d w=e^{\frac{\lambda \sqrt{\tau}}{2}} F\left(\sigma_{1}, \sigma_{2}, \tau, \lambda\right)
\end{aligned}
$$

Therefore, assuming (A.3) holds, from (A.1) and (A.4) we have

$$
e^{x-\frac{\lambda \sqrt{\tau}}{2}} \sqrt{\frac{\tau}{2 \pi}} F(0, \sigma, \tau, \lambda) \leq u^{\mathrm{BS}}(\sigma ; \tau, x, k)-\left(e^{x}-e^{k}\right)^{+} \leq e^{x+\frac{\lambda \sqrt{\tau}}{2}} \sqrt{\frac{\tau}{2 \pi}} F(0, \sigma, \tau, \lambda) .
$$


Note that $F$ in (A.2) is a monotone function, increasing in $\sigma_{2}$, decreasing in $\sigma_{1}, \tau$ and $\lambda$. In particular, for any $0 \leq \sigma_{\min } \leq \sigma_{\max }, \lambda>0, \tau_{0}>0$ and $\tau \in\left[0, \tau_{0}\right]$, we have

$$
0<F\left(\sigma_{\min }, \sigma_{2}, T, \lambda\right) \leq F\left(\sigma_{1}, \sigma_{2}, \tau, \lambda\right) \leq F\left(\sigma_{1}, \sigma_{\max }, 0, \lambda\right)<\infty, \quad \sigma_{\min } \leq \sigma_{1} \leq \sigma_{2} \leq \sigma_{\max } .
$$

The estimates in (A.5) were used by Roper and Rutkowski (2009) (see also Li (2005)) to derive the asymptotic behavior close to expiry of the Black-Scholes Call price as $\tau \downarrow 0$. Below we use (A.5) to prove two lemmas concerning the comparison, close to expiry, of two Black-Scholes prices with different volatilities.

Lemma A.1. For any $\lambda>0, \sigma_{2} \geq \sigma_{1}>0$ and $\tau_{0}>0$ there exists a constant $C \geq 1$, dependent only on $\lambda, \sigma_{1}, \sigma_{2}$ and $\tau_{0}$, such that

$$
u^{\mathrm{BS}}\left(\sigma_{2} ; \tau, x, k\right) \leq C u^{\mathrm{BS}}\left(\sigma_{1} ; \tau, x, k\right)
$$

for any $\tau \in\left[0, \tau_{0}\right]$ and $|x-k| \leq \lambda \sqrt{\tau}$.

Proof. It suffices to prove that

$$
u^{\mathrm{BS}}\left(\sigma_{2} ; \tau, x, k\right)-\left(e^{x}-e^{k}\right)^{+} \leq C\left(u^{\mathrm{BS}}\left(\sigma_{1} ; \tau, x, k\right)-\left(e^{x}-e^{k}\right)^{+}\right), \quad|x-k| \leq \lambda \sqrt{\tau}, \quad \tau \in\left[0, \tau_{0}\right] .
$$

By (A.5) we have

$$
\begin{aligned}
u^{\mathrm{BS}}\left(\sigma_{2} ; \tau, x, k\right)-\left(e^{x}-e^{k}\right)^{+} & \leq e^{x+\frac{\lambda \sqrt{\tau}}{2}} \sqrt{\frac{\tau}{2 \pi}} F\left(0, \sigma_{2}, \tau, \lambda\right) \\
& \leq e^{\lambda \sqrt{\tau_{0}}} \frac{F\left(0, \sigma_{2}, 0, \lambda\right)}{F\left(0, \sigma_{1}, \tau_{0}, \lambda\right)}\left(u^{\mathrm{BS}}\left(\sigma_{1} ; \tau, x, k\right)-\left(e^{x}-e^{k}\right)^{+}\right),
\end{aligned}
$$

where in the last inequality we used also (A.6).

Lemma A.2. For any $\lambda>0, \sigma_{2}>\sigma_{1}>0$ and $C>0$ there exists $\tau_{0}$ with $0<\tau_{0}<\frac{1}{C}$, dependent only on $\lambda, \sigma_{1}, \sigma_{2}$ and $C$, such that

$$
\begin{aligned}
u^{\mathrm{BS}}\left(\sigma_{1} ; \tau, x, k\right) & \leq(1-C \tau) u^{\mathrm{BS}}\left(\sigma_{2} ; \tau, x, k\right), \\
(1+C \tau) u^{\mathrm{BS}}\left(\sigma_{1} ; \tau, x, k\right) & \leq u^{\mathrm{BS}}\left(\sigma_{2} ; \tau, x, k\right),
\end{aligned}
$$

for any $\tau \in\left[0, \tau_{0}\right]$ and $|x-k| \leq \lambda \sqrt{\tau}$.

Proof. To establish the first inequality (A.7), we prove that

$$
u^{\mathrm{BS}}\left(\sigma_{2} ; \tau, x, k\right)-u^{\mathrm{BS}}\left(\sigma_{1} ; \tau, x, k\right) \geq C \tau u^{\mathrm{BS}}\left(\sigma_{2} ; \tau, x, k\right), \quad|x-k| \leq \lambda \sqrt{\tau}, \quad \tau \in\left[0, \tau_{0}\right] .
$$

We estimate the LHS in (A.9) using (A.1). We have

$$
\begin{aligned}
u^{\mathrm{BS}}\left(\sigma_{2} ; \tau, x, k\right)-u^{\mathrm{BS}}\left(\sigma_{1} ; \tau, x, k\right) & =\sqrt{\tau} \frac{e^{x}}{\sqrt{2 \pi}} \int_{\sigma_{1}}^{\sigma_{2}} e^{-\frac{1}{2}\left(\frac{x-k}{w \sqrt{\tau}}+\frac{w \sqrt{\tau}}{2}\right)^{2}} d w \\
& \geq \sqrt{\tau} \frac{e^{x-\frac{\lambda \sqrt{\tau}}{2}}}{\sqrt{2 \pi}} F\left(\sigma_{1}, \sigma_{2}, \tau, \lambda\right) \geq c e^{x} \sqrt{\tau}, \\
c & :=\frac{e^{-\frac{\lambda}{2 \sqrt{\tau}}}}{\sqrt{2 \pi}} F\left(\sigma_{1}, \sigma_{2}, \frac{1}{C}, \lambda\right),
\end{aligned}
$$


where in the next-to-last inequality we used (A.3) and (A.4), and in the last inequality we used (A.6) and $\tau<\frac{1}{C}$, so that $c$ is positive and independent of $\tau$. Next, once again using (A.5), we can prove the following estimate for the RHS of (A.9):

$$
u^{\mathrm{BS}}\left(\sigma_{2} ; \tau, x, k\right) \leq u^{\mathrm{BS}}\left(\sigma_{2} ; \frac{1}{C}, x, k\right) \leq e^{x}\left(1+e^{\frac{\lambda}{2 \sqrt{C}}} \sqrt{\frac{1}{2 C \pi}} F\left(0, \sigma_{2}, \frac{1}{C}, \lambda\right)\right),
$$

and therefore, for $\tau$ positive and suitably small, we have

$$
C \tau u^{\mathrm{BS}}\left(\sigma_{2} ; \tau, x, k\right) \leq c e^{x} \sqrt{\tau}
$$

for $c$ as in (A.10). This establishes the first inequality (A.7). To establish the second inequality (A.8) we have

$$
\begin{aligned}
(1+C \tau) u^{\mathrm{BS}}\left(\sigma_{1} ; \tau, x, k\right) & \leq u^{\mathrm{BS}}\left(\sigma_{1} ; \tau, x, k\right)+C \tau u^{\mathrm{BS}}\left(\sigma_{2} ; \tau, x, k\right) \\
& \leq(1-C \tau) u^{\mathrm{BS}}\left(\sigma_{2} ; \tau, x, k\right)+C \tau u^{\mathrm{BS}}\left(\sigma_{2} ; \tau, x, k\right)=u^{\mathrm{BS}}\left(\sigma_{2} ; \tau, x, k\right),
\end{aligned}
$$

where we have used (A.7) in the last inequality. This concludes the proof.

\section{B Faà di Bruno's formula and Bell polynomials}

Here we briefly recall the well known Faà di Bruno's formula (see Riordan (1946) and Johnson (2002)), more precisely, its Bell polynomial version. Let $f$ and $g$ be two $C^{\infty}$ real-valued functions on $\mathbb{R}$. The following representation holds:

$$
\frac{\mathrm{d}^{n}}{\mathrm{~d} x^{n}} f(g(x))=\sum_{h=1}^{n} f^{(h)}(g(x)) \cdot \mathbf{B}_{n, h}\left(\frac{\mathrm{d}}{\mathrm{d} x} g(x), \frac{\mathrm{d}^{2}}{\mathrm{~d} x^{2}} g(x), \cdots, \frac{\mathrm{d}^{n-h+1}}{\mathrm{~d} x^{n-h+1}} g(x)\right), \quad n \geq 1,
$$

with $\mathbf{B}_{n, h}$ being the family of the Bell polynomials defined as

$$
\mathbf{B}_{n, h}(z)=\sum \frac{n !}{j_{1} ! j_{2} ! \cdots j_{n-h+1} !}\left(\frac{z_{1}}{1 !}\right)^{j_{1}}\left(\frac{z_{2}}{2 !}\right)^{j_{2}} \cdots\left(\frac{z_{n-h+1}}{(n-h+1) !}\right)^{j_{n-h+1}}, \quad 1 \leq h \leq n,
$$

where the sum is taken over all sequences $j_{1}, j_{2}, \cdots, j_{n-h+1}$ of non-negative integers such that

$$
j_{1}+j_{2}+\cdots+j_{n-h+1}=h, \quad \text { and } \quad j_{1}+2 j_{2}+\cdots+(n-h+1) j_{n-h+1}=n .
$$

\section{Implied volatility expressions}

In this appendix we assume a time-homogeneous diffusion and use the Taylor series expansion of $\mathcal{A}$ as in Example 2.3 with $(\bar{x}, \bar{y})=\left(X_{0}, Y_{0}\right):=(x, y)$. With $\mathcal{A}$ given by (2.4), we introduce the notation

$$
\eta_{i, j}=\frac{\partial_{x}^{i} \partial_{y}^{j} \eta(\bar{x}, \bar{y})}{i ! j !}, \quad \eta \in\{a, b, c, f\} .
$$

and we compute, explicitly (below $\tau$ is time to maturity)

$$
\sigma_{0}=\sqrt{2 a_{0,0}}, \quad \sigma_{1}=\sigma_{1,0}+\sigma_{0,1}, \quad \sigma_{2}=\sigma_{2,0}+\sigma_{1,1}+\sigma_{0,2},
$$


where

$$
\sigma_{1,0}=\left(\frac{a_{1,0}}{2 \sigma_{0}}\right)(k-x), \quad \sigma_{0,1}=\tau\left(\frac{a_{0,1}\left(c_{0,0}+2 f_{0,0}\right)}{4 \sigma_{0}}\right)+\left(\frac{a_{0,1} c_{0,0}}{2 \sigma_{0}^{3}}\right)(k-x),
$$

and

$$
\begin{aligned}
& \sigma_{2,0}=\tau\left(\frac{1}{12} \sigma_{0} a_{2,0}-\frac{a_{1,0}^{2}}{8 \sigma_{0}}\right)+\tau^{2}\left(-\frac{1}{96} \sigma_{0} a_{1,0}^{2}\right)+\left(\frac{2 \sigma_{0}^{2} a_{2,0}-3 a_{1,0}^{2}}{12 \sigma_{0}^{3}}\right)(k-x)^{2}, \\
& \sigma_{1,1}=\frac{\tau}{12 \sigma_{0}^{3}}\left(\sigma_{0}^{2} a_{1,1} c_{0,0}+a_{0,1}\left(a_{1,0} c_{0,0}-2 \sigma_{0}^{2} c_{1,0}\right)\right)+\frac{\tau^{2}}{48 \sigma_{0}}\left(-a_{0,1} a_{1,0} c_{0,0}\right) \\
& \quad+\frac{\tau}{24 \sigma_{0}^{3}}\left(2 \sigma_{0}^{2} a_{1,1}\left(c_{0,0}+2 f_{0,0}\right)+a_{0,1}\left(2 \sigma_{0}^{2}\left(c_{1,0}+2 f_{1,0}\right)-5 a_{1,0}\left(c_{0,0}+2 f_{0,0}\right)\right)\right)(k-x) \\
& \quad+\frac{1}{6 \sigma_{0}^{5}}\left(\sigma_{0}^{2} a_{1,1} c_{0,0}+a_{0,1}\left(\sigma_{0}^{2} c_{1,0}-5 a_{1,0} c_{0,0}\right)\right)(k-x)^{2}, \\
& \sigma_{0,2}=\frac{\tau}{24 \sigma_{0}^{5}}\left(4 \sigma_{0}^{2} a_{0,2}\left(3 \sigma_{0}^{2} b_{0,0}-c_{0,0}^{2}\right)+a_{0,1}\left(a_{0,1}\left(9 c_{0,0}^{2}-8 \sigma_{0}^{2} b_{0,0}\right)-4 \sigma_{0}^{2} c_{0,0} c_{0,1}\right)\right) \\
& \quad+\frac{\tau^{2}}{24 \sigma_{0}^{3}}\left(a_{0,1}\left(-2 \sigma_{0}^{2} a_{0,1} b_{0,0}+c_{0,0}\left(\sigma_{0}^{2}\left(c_{0,1}+2 f_{0,1}\right)-3 a_{0,1} f_{0,0}\right)\right)\right. \\
&\left.\quad+a_{0,1} f_{0,0}\left(2 \sigma_{0}^{2}\left(c_{0,1}+2 f_{0,1}\right)-3 a_{0,1} f_{0,0}\right)+\sigma_{0}^{2} a_{0,2}\left(c_{0,0}+2 f_{0,0}\right)^{2}\right) \\
& \quad+\frac{\tau}{24 \sigma_{0}^{5}}\left(a_{0,1}\left(c_{0,0}\left(4 \sigma_{0}^{2}\left(c_{0,1}+f_{0,1}\right)-18 a_{0,1} f_{0,0}\right)-9 a_{0,1} c_{0,0}^{2}+4 \sigma_{0}^{2} c_{0,1} f_{0,0}\right)\right. \\
&\left.\quad+4 \sigma_{0}^{2} a_{0,2} c_{0,0}\left(c_{0,0}+2 f_{0,0}\right)\right)(k-x) \\
& \quad+\frac{1}{12 \sigma_{0}^{7}}\left(a_{0,1}\left(a_{0,1}\left(4 \sigma_{0}^{2} b_{0,0}-9 c_{0,0}^{2}\right)+2 \sigma_{0}^{2} c_{0,0} c_{0,1}\right)+2 \sigma_{0}^{2} a_{0,2} c_{0,0}^{2}\right)(k-x)^{2},
\end{aligned}
$$

Higher order terms are too long to reasonably include in this text. However, $\sigma_{3}$ and (for local volatility models) $\sigma_{4}$ can be computed easily using the Mathematica code provided free of charge on the authors' website.

http://explicitsolutions. wordpress.com

\section{References}

Alexander, C. and L. Nogueira (2004). Stochastic local volatility. Proceedings of the Second IASTED Int. Conf. on Financial Engineering and Applications, Cambridge MA, USA, 136-141.

Andersen, L. B. and V. V. Piterbarg (2007). Moment explosions in stochastic volatility models. Finance and Stochastics 11(1), $29-50$.

Antonov, A. and M. Spector (2012, march). Advanced analytics for the sabr model. SSRN.

Baldeaux, J. and A. Badran (2012). Consistent modeling of vix and equity derivatives using a $3 / 2$ plus jumps model. arXiv preprint arXiv:1203.5903.

Benhamou, E., E. Gobet, and M. Miri (2010). Time dependent Heston model. SIAM J. Financial Math. 1(1), $289-325$.

Berestycki, H., J. Busca, and I. Florent (2002). Asymptotics and calibration of local volatility models. Quantitative finance 2(1), $61-69$.

Berestycki, H., J. Busca, and I. Florent (2004). Computing the implied volatility in stochastic volatility models. Communications on Pure and Applied Mathematics 57(10), 1352-1373. 
Bompis, R. and E. Gobet (2012). Asymptotic and non asymptotic approximations for option valuation. in Recent Developments in Computational Finance:Foundations, Algorithms and Applications, T. Gerstner and P. Kloeden (Ed.), World Scientific Publishing Company, 1-80.

Clark, I. (2010). Foreign Exchange Option Pricing: A Practitioner's Guide. Chichester: Wiley.

Cox, J. (1975). Notes on option pricing I: Constant elasticity of diffusions. Unpublished draft, Stanford University. A revised version of the paper was published by the Journal of Portfolio Management in 1996.

Drimus, G. G. (2012). Options on realized variance by transform methods: a non-affine stochastic volatility model. Quant. Finance 12(11), 1679-1694.

Duffie, D., J. Pan, and K. Singleton (2000). Transform analysis and asset pricing for affine jump-diffusions. Econometrica 68(6), $1343-1376$.

Ewald, C.-O. (2005). Local volatility in the Heston model: a Malliavin calculus approach. J. Appl. Math. Stoch. Anal. (3), $307-322$.

Forde, M. and A. Jacquier (2009). Small-time asymptotics for implied volatility under the heston model. International Journal of Theoretical and Applied Finance 12(06), 861-876.

Forde, M. and A. Jacquier (2011). Small-time asymptotics for an uncorrelated local-stochastic volatility model. Applied Mathematical Finance 18(6), 517-535.

Forde, M., A. Jacquier, and R. Lee (2012). The small-time smile and term structure of implied volatility under the heston model. SIAM Journal on Financial Mathematics 3(1), 690-708.

Fouque, J.-P., M. Lorig, and R. Sircar (2012). Second order multiscale stochastic volatility asymptotics: Stochastic terminal layer analysis and calibration. ArXiv preprint arXiv:1209.0697.

Fouque, J.-P., G. Papanicolaou, R. Sircar, and K. Solna (2011). Multiscale stochastic volatility for equity, interest rate, and credit derivatives. Cambridge: Cambridge University Press.

Friedman, A. (1964). Partial differential equations of parabolic type. Englewood Cliffs, N.J.: Prentice-Hall Inc.

Friz, P. K., S. Gerhold, and M. Yor (2013). How to make Dupire's local volatility work with jumps. arXiv preprint1302.5548.

Gao, K. and R. Lee (2014). Asymptotics of implied volatility to arbitrary order. to appear in Finance and Stochastics.

Gatheral, J., E. P. Hsu, P. Laurence, C. Ouyang, and T.-H. Wang (2012). Asymptotics of implied volatility in local volatility models. Math. Finance 22(4), 591-620.

Hagan, P., D. Kumar, A. Lesniewski, and D. Woodward (2002). Managing smile risk. Wilmott Magazine 1000, 84-108.

Hagan, P. and D. Woodward (1999). Equivalent black volatilities. Applied Mathematical Finance 6(3), $147-157$.

Henry-Labordère, P. (2005). A general asymptotic implied volatility for stochastic volatility models. eprint arXiv:condmat/0504317.

Henry-Labordère, P. (2009). Analysis, geometry, and modeling in finance: Advanced methods in option pricing, Volume 13. Chapman \& Hall.

Henry-Labordere, P. (2009). Calibration of local stochastic volatility models to market smiles: A Monte-Carlo approach. RISK (September), 112-117.

Heston, S. (1993). A closed-form solution for options with stochastic volatility with applications to bond and currency options. Rev. Financ. Stud. 6(2), 327-343.

Ikeda, N. and S. Watanabe (1989). Stochastic differential equations and diffusion processes (Second ed.), Volume 24 of NorthHolland Mathematical Library. Amsterdam: North-Holland Publishing Co. 
Jacquier, A. and M. Lorig (2013). The smile of certain lévy-type models. ArXiv preprint arXiv:120\%.1630.

Jacquier, A. and M. Lorig (2014). From characteristic functions to implied volatility expansions. To appear in: Advances in Applied Probability.

Johnson, W. P. (2002). The curious history of Faà di Bruno's formula. Amer. Math. Monthly 109(3), 217-234.

Lee, R. W. (2004). The moment formula for implied volatility at extreme strikes. Mathematical Finance 14(3), 469-480.

Lewis, A. (2007). Geometries and smile asymptotics for a class of stochastic volatility models.

Li, S. (2005). A new formula for computing implied volatility. Appl. Math. Comput. 170(1), 611-625.

Lipton, A. (2002). The vol smile problem. Risk (February), 61-65.

Lorig, M. (2013). The exact smile of certain local volatility models. Quantitative Finance 13(6), 897-905.

Lorig, M., S. Pagliarani, and A. Pascucci (2013). Analytical expansions for parabolic equations. ArXiv preprint arXiv:1312.3314.

Lorig, M., S. Pagliarani, and A. Pascucci (2014). A family of density expansions for Lévy-type processes with default. To appear in: Annals of Applied Probability.

Obloj, J. (2008, 5). Fine-tune your smile: Correction to Hagan et al. Wilmott Magazine.

Pagliarani, S. and A. Pascucci (2012). Analytical approximation of the transition density in a local volatility model. Cent. Eur. J. Math. 10(1), 250-270.

Pagliarani, S. and A. Pascucci (2014). Asymptotic expansions for degenerate parabolic equations. SSRN.

Pagliarani, S., A. Pascucci, and C. Riga (2013). Adjoint expansions in local Lévy models. SIAM J. Financial Math. 4, $265-296$.

Pascucci, A. (2011). PDE and martingale methods in option pricing, Volume 2 of Bocconi ES Springer Series. Milan: Springer.

Riordan, J. (1946). Derivatives of composite functions. Bull. Amer. Math. Soc. 52, 664-667.

Roper, M. and M. Rutkowski (2009). On the relationship between the call price surface and the implied volatility surface close to expiry. International Journal of Theoretical and Applied Finance 12(04), 427-441.

Watanabe, S. (1987). Analysis of Wiener functionals (Malliavin calculus) and its applications to heat kernels. Ann. Probab. 15(1), 1-39. 

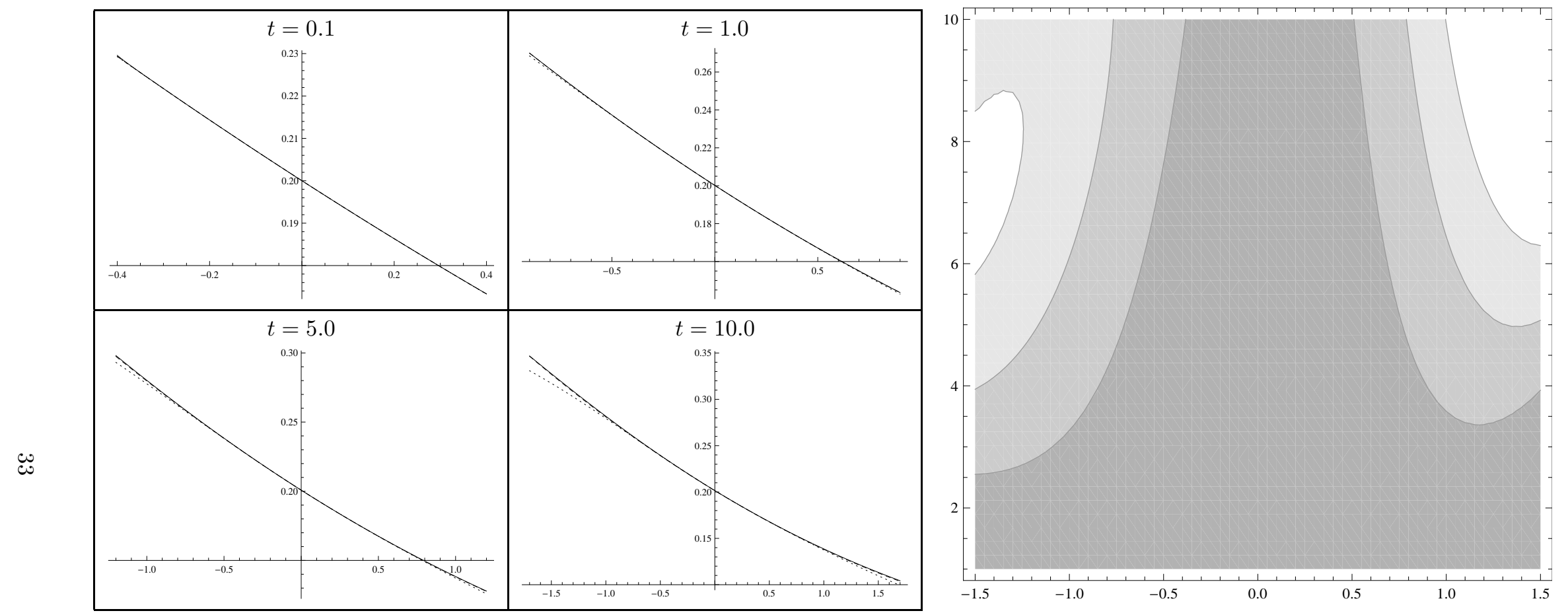

Figure 1: LEFT: Implied volatility in the CEV model (5.1) is plotted as a function of log-moneyness $(k-x)$ for four different maturities $t$. The solid line corresponds to the implied volatility $\sigma$ obtained by computing the exact price $u$ using (5.3) and then by solving (3.3) numerically. The dashed line (which is nearly indistinguishable from the solid line) corresponds to our third order implied volatility approximation $\bar{\sigma}_{3}$, which we compute by summing the terms in (5.2). The dotted line corresponds to the implied volatility expansion $\sigma^{\mathrm{HW}}$ of Hagan and Woodward (1999), which is computed using (5.4). RIGHT: We plot the absolute value of the relative error $\left|\bar{\sigma}_{3}-\sigma\right| / \sigma$ of our third order implied volatility approximation as a function of $\log$-moneyness $(k-x)$ and maturity $t$. The horizontal axis represents log-moneyness $(k-x)$ and the vertical axis represents maturity $t$. Ranging from darkest to lightest, the regions above represent relative errors of $<0.3 \%, 0.3 \%$ to $0.6 \%, 0.6 \%$ to $0.9 \%$ and $>0.9 \%$. We use the following parameters: $\beta=0.3, \delta=0.2, x=0.0$. 

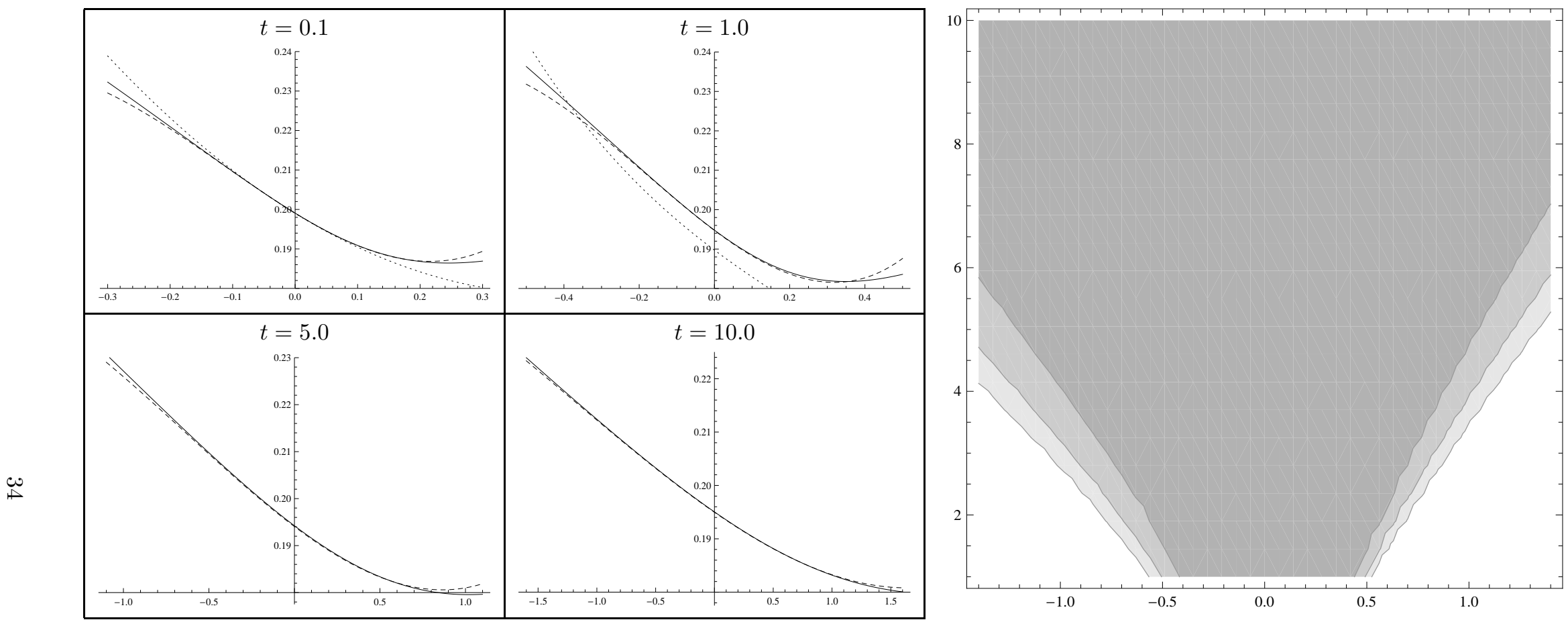

Figure 2: LEFT: Implied volatility the Heston model (5.5) is plotted as a function of log-moneyness $(k-x)$ for four different maturities $t$. The solid line corresponds to the implied volatility $\sigma$, obtained by computing the exact price $u$ using (5.7) and then by solving (3.3) numerically. The dashed line corresponds to our third order implied volatility approximation $\bar{\sigma}_{3}$, which we compute by summing the terms in (5.6) (note: $\sigma_{3}$ does not appear in the text). The dotted line (which only appears for the shortest two maturities) corresponds to the implied volatility expansion $\sigma^{\mathrm{FJL}}$ of Forde et al. (2012); it is computed using (5.8). Note that the dotted line does not appear in the plots for the two largest maturities. RIGHT: We plot the absolute value of the relative error $\left|\bar{\sigma}_{3}-\sigma\right| / \sigma$ of our third order implied volatility approximation as a function of log-moneyness $(k-x)$ and maturity $t$. The horizontal axis represents log-moneyness $(k-x)$ and the vertical axis represents maturity $t$. Ranging from darkest to lightest, the regions above represent relative errors of $<1 \%, 1 \%$ to $2 \%, 2 \%$ to $3 \%$ and $>3 \%$. We use the parameters given in Forde et al. $(2012): \kappa=1.15, \theta=0.04, \delta=0.2$, $\rho=-0.40 x=0.0, y=\log \theta$. 

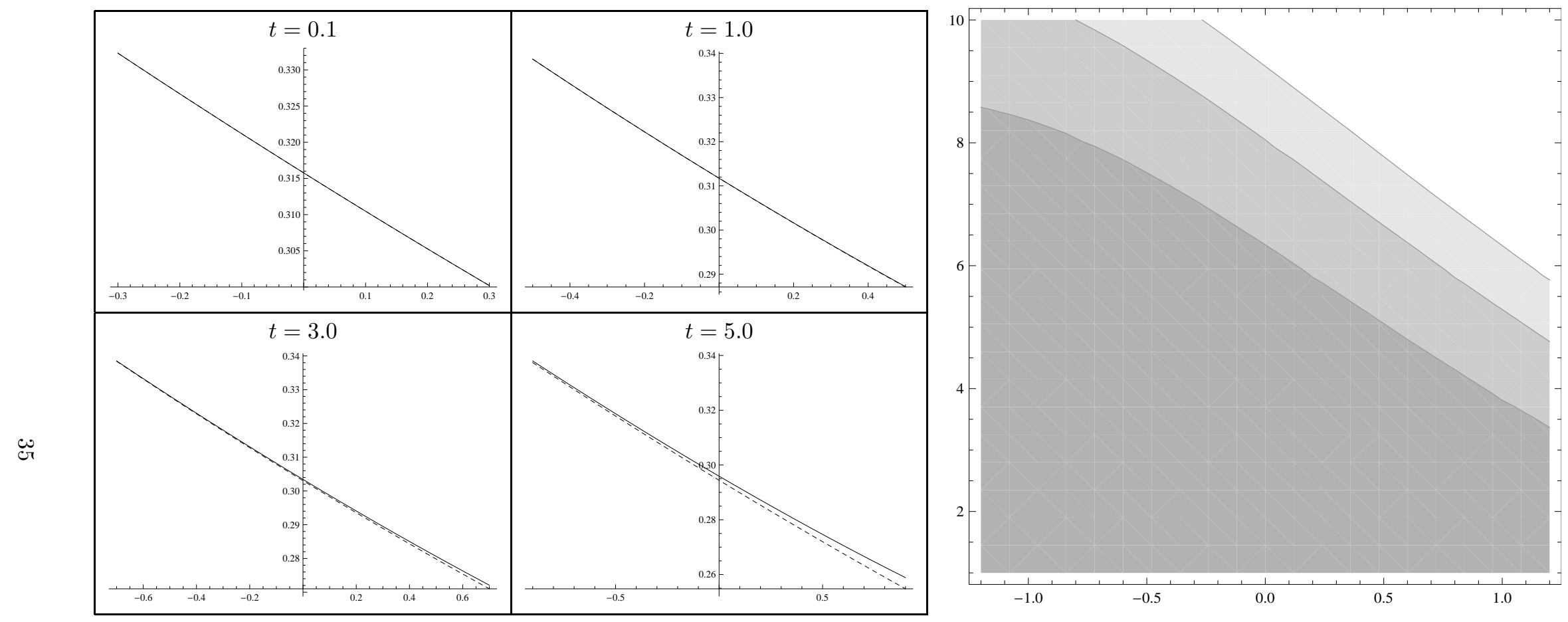

Figure 3: LEFT: Implied volatility in the $3 / 2$ stochastic volatility model (5.9) is plotted as a function of log-moneyness $(k-x)$ for four different maturities $t$. The solid line corresponds to the implied volatility $\sigma$, obtained by computing the exact price $u$ using (5.12) and then by solving (3.3) numerically. The dashed line corresponds to our third order implied volatility approximation $\bar{\sigma}_{3}$, which we compute by summing the terms in (5.10). RIGHT: We plot the absolute value of the relative error $\left|\bar{\sigma}_{3}-\sigma\right| / \sigma$ of our third order implied volatility approximation as a function of log-moneyness $(k-x)$ and maturity $t$. The horizontal axis represents log-moneyness $(k-x)$ and the vertical axis represents maturity $t$. Ranging from darkest to lightest, the regions above represent relative errors of $<1 \%, 1 \%$ to $2 \%, 2 \%$ to $3 \%$ and $>3 \%$. We use the following parameters: $\kappa=0.25, \theta=0.1$, $\delta=0.8, \rho=-0.85 x=0.0, y=\log \theta$. 

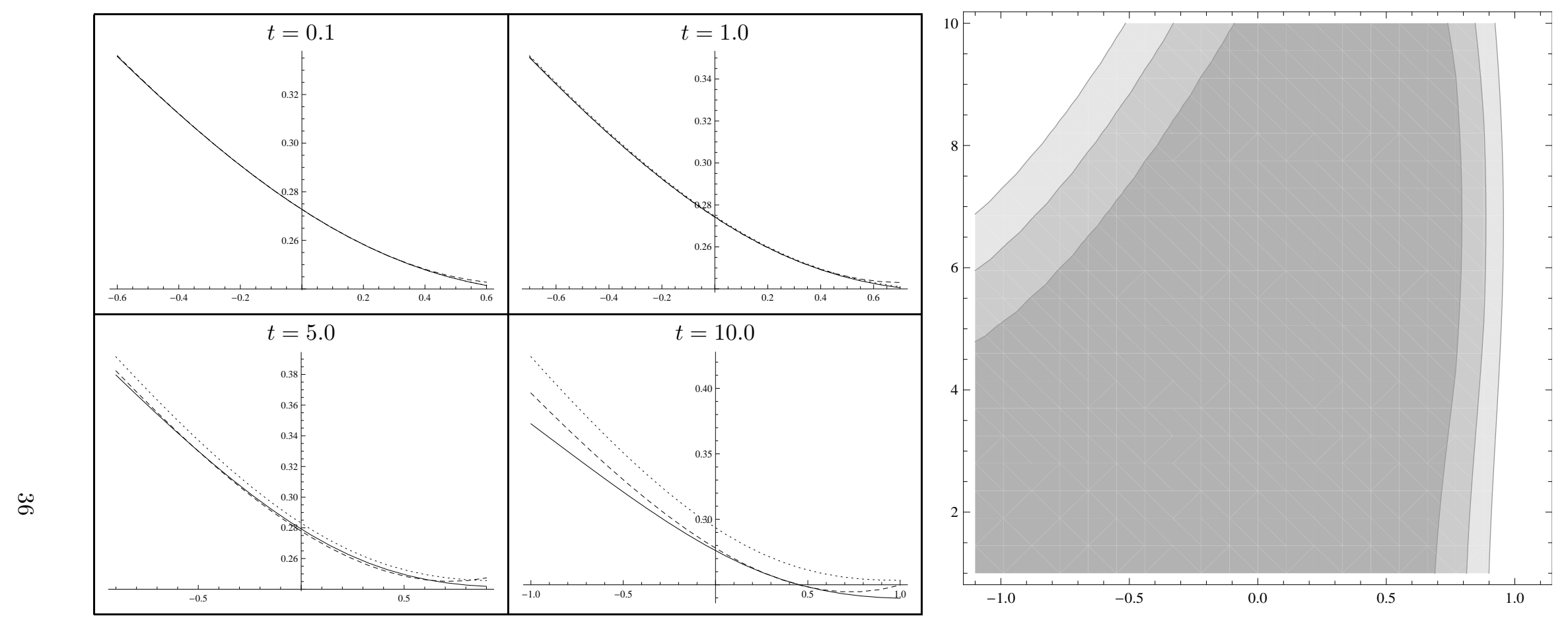

Figure 4: LEFT: Implied volatility in the SABR model (5.13) is plotted as a function of log-moneyness $(k-x)$ for four different maturities $t$. The solid line corresponds to the implied volatility $\sigma$, obtained by computing the exact price $u$ using (5.15) and then by solving (3.3) numerically. The dashed line corresponds to our third order implied volatility approximation $\bar{\sigma}_{3}$, which we compute using (5.14). The dotted line corresponds to the implied volatility expansion $\sigma^{\mathrm{HKLW}}$ of Hagan et al. (2002), which is computed using (5.16). For the two shortest maturities, both implied volatility expansions $\bar{\sigma}_{3}$ and $\sigma^{\mathrm{HKLW}}$ provide an excellent approximation of the true implied volatility $\sigma$. However, for the two longest maturities, it is clear that our third order expansion $\bar{\sigma}_{3}$ provides a better approximation to the true implied volatility $\sigma$ than does the implied volatility expansion $\sigma^{\mathrm{HKLW}}$ of Hagan et al. (2002). RIGHT: We plot the absolute value of the relative error $\left|\bar{\sigma}_{3}-\sigma\right| / \sigma$ of our third order implied volatility approximation as a function of log-moneyness $(k-x)$ and maturity $t$. The horizontal axis represents log-moneyness $(k-x)$ and the vertical axis represents maturity t. Ranging from darkest to lightest, the regions above represent relative errors of $<1 \%, 1 \%$ to $2 \%, 2 \%$ to $3 \%$ and $>3 \%$. We use the following parameters: $\beta=0.4, \delta=0.25, \rho=0.0, x=0.0, y=-1.3$. 